\title{
REFINED ANALYTIC TORSION AS ANALYTIC FUNCTION ON THE REPRESENTATION VARIETY AND APPLICATIONS
}

\author{
MAXIM BRAVERMAN AND BORIS VERTMAN
}

\begin{abstract}
We prove that refined analytic torsion on a manifold with boundary is a weakly holomorphic section of the determinant line bundle over the representation variety. As a fundamental application we establish a gluing formula for refined analytic torsion on connected components of the complex representation space which contain a unitary point. Finally we provide a new proof of Brüning-Ma gluing formula for the Ray-Singer torsion associated to a non-Hermitian connection. Our proof is quite different from the one given by Brüning and Ma and uses a temporal gauge transformation.
\end{abstract}

\section{Contents}

1. Introduction and statement of the main results 1

2. Refined analytic torsion on manifolds with boundary 3

3. Holomorphic structure on the determinant line bundle 9

4. The graded determinant as a holomorphic function 12

5. Refined analytic torsion as a holomorphic section 18

6. Gluing formula for refined analytic torsion 25

7. Gluing formula for Ray-Singer analytic torsion 30

8. Appendix: Temporal Gauge Transformation 32

References $\quad 35$

\section{INTRODUCTION AND STATEMENT OF THE MAIN RESUlTS}

The Ray-Singer conjecture has been formulated in the seminal paper of Ray and Singer [38] and proved independently by Cheeger [17] and Müller [35] for unitary representations. Its importance stems from the fact that as in the Atiyah-Singer index theorem, it equates analytic with combinatorial quantities, the analytic Ray-Singer and the combinatorial Reidemeister torsions.

Date: This document was compiled on: September 18, 2018. 
By construction, both the analytic Ray-Singer and the combinatorial Reidemeister torsions provide canonical norms on the determinant line of cohomology. There have been various approaches to obtain a canonical construction of analytic and Reidemeister torsions as elements instead of norms of the determinant line of the cohomology. These constructions seek to refine the notion of analytic and Reidemeister torsion norms on that determinant line, which basically corresponds to fixing a complex phase in the family of complex vectors of length one.

In case of the Reidemeister torsion this has been done by Farber and Turaev [18] and [19]. Refinement of analytic torsion has been studied by the first author jointly with Kappeler in [7] and [5], as well as by Burghelea and Haller in [15] and [16]. Both notions have subsequently been compared by the first author jointly with Kappeler in [6]. An extension of refined analytic torsion to manifolds with boundary has been undertaken by the second author [44] and Lee and Huang [30] in two different independent constructions. Recently, Lee and Huang also compared the two notions of refined analytic torsion on manifolds with boundary in [32].

The fundamental property of the refined analytic and Farber-Turaev torsions is that they define weakly holomorphic functions on the complex representation space (see [24, p. 148] for definition of a weakly holomorphic function). In particular, its restriction to the regular part of the representation space is holomorphic. The main purpose of the present discussion is an extension of this result to the refined analytic torsion on manifolds with boundary, introduced by the second author in [44]. As a consequence we establish the gluing property of refined analytic torsion on connected components of the representation variety that contain a unitary point.

The gluing formula for refined analytic torsion may be used to prove a gluing result for the Ray-Singer torsion norm for certain non-unitary representations path-connected to a unitary element. However we chose to devote the final two sections of the present paper to an alternative proof of the gluing property for the Ray Singer analytic torsion for non-unitary representations, which is stronger since we do not single out connected components without unitary elements.

The Ray-Singer theorem has been extended to unimodular representations by Müller [36]. In case of a general non-unitary representation, the quotient of the analytic and Reidemeister torsion norms admits additional correction terms which have been studied by Bismut and 
Zhang in [2]. In a separate discussion [9], the authors employ analyticity of refined analytic torsion to provide an alternative derivation of the Bismut-Zhang correction terms for the connected components of unitary points in the representation variety.

Both the analytic and combinatorial Reidemeister torsions make sense on compact manifolds with boundary after posing relative or absolute boundary conditions. The gluing property of the analytic torsion, which is foremost a spectral invariant, is striking and has been proved by Lück [34], Vishik [46] and generalized by Lesch [33], under the assumption of product metric structures and unitary representations.

The anomaly of analytic torsion on a compact manifold with boundary with a non-unitary representation and general metric structures near the boundary, has been studied by Brüning and Ma in [11]. Recently, Brüning and Ma established in a follow-up paper [12] the gluing formula for analytic torsion in case of a non-unitary representation, generalizing previous results in [34] and [46]. We present an alternative proof of a result by Brüning-Ma [12] by a temporal gauge transformation argument.

\section{REFINED ANALYTIC TORSION ON MANIFOLDS WITH BOUNDARY}

This section reviews the construction by the second author [44].

2.1. The flat vector bundle induced by a representation. Let $\left(M^{m}, g\right)$ be a compact oriented odd-dimensional Riemannian manifold with boundary $\partial M$. Consider a complex representation $\alpha$ of the fundamental group $\pi_{1}=\pi_{1}(M)$ on $\mathbb{C}^{n}$. Let $\left(E_{\alpha}, \nabla_{\alpha}, h_{\alpha}^{E}\right)$ be the induced flat complex vector bundle over $M$ with monodromy equal to $\alpha$ and no canonical choice of $h_{\alpha}^{E}$ in case $\alpha$ is not unitary.

The flat covariant derivative $\nabla_{\alpha}$ acts on sections $\Gamma\left(E_{\alpha}\right)$ and extends by Leibniz rule to a twisted differential on $E_{\alpha}$-valued differential forms $\Omega_{0}^{*}\left(M, E_{\alpha}\right)$, where the lower index refers to compact support in the open interior of $M$. This defines the twisted de Rham complex $\left(\Omega_{0}^{*}\left(M, E_{\alpha}\right), \nabla_{\alpha}\right)$. The metrics $\left(g, h_{\alpha}^{E}\right)$ induce an $L^{2}$-inner product on $\Omega_{0}^{*}\left(M, E_{\alpha}\right)$. We denote the $L^{2}-$ completion of $\Omega_{0}^{*}\left(M, E_{\alpha}\right)$ by $L_{*}^{2}\left(M, E_{\alpha}\right)$.

Throughout this section the representation $\alpha$ is fixed and we omit the lower index $\alpha$ in the notation of $\left(E_{\alpha}, \nabla_{\alpha}, h_{\alpha}^{E}\right)$ in most of the discussion.

Next we introduce the notion of the dual covariant derivative $\nabla^{\prime}$. It is defined by requiring for all $u, v \in \Gamma(E)$ and $X \in \Gamma(T M)$

$$
d h^{E}(u, v)[X]=h^{E}\left(\nabla_{X} u, v\right)+h^{E}\left(u, \nabla_{X}^{\prime} v\right) .
$$


In the special case that $\alpha$ is unitary, the dual $\nabla^{\prime}$ and the original covariant derivative $\nabla$ coincide. As before, the dual $\nabla^{\prime}$ gives rise to a twisted de Rham complex $\left(\Omega_{0}^{*}(M, E), \nabla^{\prime}\right)$.

2.2. Hilbert complexes. For any differential operator $P$ acting on $\Omega_{0}^{*}(M, E)$, we denote by $P_{\min }$ its minimal graph-closed extension in $L_{*}^{2}(M, E)$. The maximal closed extension is defined by $P_{\max }:=\left(P_{\min }^{t}\right)^{*}$. By Brüning and Lesch [10, Lemma 3.1], the extensions define Hilbert complexes $\left(\mathcal{D}_{\text {min }}, \nabla_{\text {min }}\right)$, where $\mathcal{D}_{\text {min }}:=\mathcal{D}\left(\nabla_{\text {min }}\right)$, and $\left(\mathcal{D}_{\max }, \nabla_{\text {max }}\right)$, where $\mathcal{D}_{\text {max }}:=\mathcal{D}\left(\nabla_{\text {max }}\right)$. The Laplace operators, associated to these Hilbert complexes are respectively defined as

$$
\begin{aligned}
\triangle_{\text {rel }} & :=\nabla_{\min }^{*} \nabla_{\text {min }}+\nabla_{\text {min }} \nabla_{\text {min }}^{*}, \\
\triangle_{\text {abs }}: & =\nabla_{\text {max }}^{*} \nabla_{\max }+\nabla_{\max } \nabla_{\text {max }}^{*} .
\end{aligned}
$$

Similar definitions hold for the dual connection $\nabla^{\prime}$ and for the Laplace operators $\triangle_{\text {rel }}^{\prime}$ and $\triangle_{\text {abs }}^{\prime}$ of the Hilbert complexes $\left(\mathcal{D}_{\text {min }}^{\prime}, \nabla_{\text {min }}^{\prime}\right)$ and $\left(\mathcal{D}_{\max }^{\prime}, \nabla_{\max }^{\prime}\right)$ respectively. The difference $\left(\nabla-\nabla^{\prime}\right)$ is a bounded endomorphism valued operator and hence the equality of domains

$$
\mathcal{D}_{\text {min }}=\mathcal{D}_{\text {min }}^{\prime}, \quad \mathcal{D}_{\text {max }}=\mathcal{D}_{\text {max }}^{\prime} .
$$

The following theorem, compare [44, Theorem 3.2], summarizes the classical de Rham theorem on manifolds with boundary, cf. [38, Remark after Proposition 4.2] and [10, Theorem 4.1]; strong ellipticity of the corresponding Laplace operators follows from [22, Lemma 1.11.1].

Theorem 2.3. The Hilbert complexes $\left(\mathcal{D}_{\min }, \nabla_{\min }\right)$ and $\left(\mathcal{D}_{\max }, \nabla_{\max }\right)$ are Fredholm and the associated Laplacians $\triangle_{\text {rel }}$ and $\triangle_{\mathrm{abs}}$ are strongly elliptic. The cohomologies $H^{*}(M, \partial M, E)$ and $H^{*}(M, E)$ of the Fredholm complexes $\left(\mathcal{D}_{\min }, \nabla_{\min }\right)$ and $\left(\mathcal{D}_{\max }, \nabla_{\max }\right)$, respectively, can be computed from the following smooth subcomplexes,

$$
\begin{array}{ll}
\left(\Omega_{\min }^{*}(M, E), \nabla\right), & \Omega_{\min }^{*}(M, E):=\left\{\omega \in \Omega^{*}(M, E) \mid \iota^{*}(\omega)=0\right\}, \\
\left(\Omega_{\max }^{*}(M, E), \nabla\right), & \Omega_{\max }^{*}(M, E):=\Omega^{*}(M, E),
\end{array}
$$

respectively, where $\iota: \partial M \hookrightarrow M$ denotes the natural inclusion of the boundary. Corresponding statement holds also for the complexes associated to the dual connection $\nabla^{\prime}$.

2.4. The chirality operator. The Riemannian metric $g$ and a fixed orientation on $M$ define the Hodge star operator $*$ and the chirality operator $(r:=(m+1) / 2)$

$$
\Gamma:=i^{r}(-1)^{\frac{k(k+1)}{2}} *: \Omega^{k}(M, E) \rightarrow \Omega^{m-k}(M, E) .
$$


This operator extends to a self-adjoint involution on $L_{*}^{2}(M, E)$. The following properties of $\Gamma$ are essential for the construction below, cf. [44].

Lemma 2.5. The self-adjoint involution $\Gamma$ on $L_{*}^{2}(M, E)$ maps $\mathcal{D}\left(\nabla_{\min }\right)$ to $\mathcal{D}\left(\nabla_{\max }^{* *}\right)$, and $\mathcal{D}\left(\nabla_{\max }\right)$ to $\mathcal{D}\left(\nabla_{\min }^{*}\right)$. With $\Gamma$ restricted to appropriate domains, we have

$$
\Gamma \nabla_{\min } \Gamma=\nabla_{\max }^{*}, \quad \Gamma \nabla_{\max } \Gamma=\nabla_{\min }^{*} .
$$

Definition 2.6. We introduce the doubled Hilbert complexes

$$
\begin{aligned}
& (\widetilde{\mathcal{D}}, \mathbb{D}):=\left(\mathcal{D}_{\text {min }}, \nabla_{\text {min }}\right) \oplus\left(\mathcal{D}_{\text {max }}, \nabla_{\text {max }}\right), \\
& \left(\widetilde{\mathcal{D}}^{\prime}, \mathbb{D}^{\prime}\right):=\left(\mathcal{D}_{\text {min }}^{\prime}, \nabla_{\text {min }}^{\prime}\right) \oplus\left(\mathcal{D}_{\text {max }}^{\prime}, \nabla_{\text {max }}^{\prime}\right) .
\end{aligned}
$$

Similar to (2.2), we have the equality of domains

$$
\mathcal{D}(\mathbb{D})=\mathcal{D}\left(\mathbb{D}^{\prime}\right), \quad \mathcal{D}\left(\mathbb{D}^{*}\right)=\mathcal{D}\left(\mathbb{D}^{*}\right) .
$$

The self-adjoint involution $\Gamma$ gives rise to the "chirality operator"

$$
\mathbb{G}:=\left(\begin{array}{cc}
0 & \Gamma \\
\Gamma & 0
\end{array}\right) \text { on } L_{*}^{2}(M, E) \oplus L_{*}^{2}(M, E) .
$$

An immediate consequence of Lemma 2.5 is the following

Proposition 2.7. The chirality operator $\mathbb{G}$ acts as

$$
\left.\mathbb{G}\right|_{\mathcal{D}(\mathbb{D})}: \mathcal{D}(\mathbb{D}) \rightarrow \mathcal{D}\left(\mathbb{D}^{*}\right),\left.\quad \mathbb{G}\right|_{\mathcal{D}\left(\mathbb{D}^{*}\right)}: \mathcal{D}\left(\mathbb{D}^{*}\right) \rightarrow \mathcal{D}(\mathbb{D}) .
$$

Moreover we have the relation $\mathbb{G} \mathbb{D}=\mathbb{D}^{*} \mathbb{G}$.

2.8. The odd signature operator. We now apply the concepts of [7] to our new setup and define the odd-signature operator of the Hilbert complex $(\widetilde{\mathcal{D}}, \mathbb{D})$ by

$$
\mathcal{B}:=\mathbb{G D}+\mathbb{D} \mathbb{G}, \quad \mathcal{D}(\mathcal{B})=\mathcal{D}(\mathbb{D}) \cap \mathcal{D}\left(\mathbb{D}^{*}\right) .
$$

By [44] the odd signature operator $\mathcal{B}$ is strongly elliptic with discrete spectrum and an Agmon angle $\theta \in(-\pi, 0)$.

2.9. Spectral decomposition. Consider for any $\lambda \geq 0$ the spectral projection of $\mathcal{B}^{2}$ onto eigenspaces with eigenvalues of absolute value in the interval $[0, \lambda]$ :

$$
\Pi_{\mathcal{B}^{2},[0, \lambda]}:=\frac{i}{2 \pi} \int_{\gamma(\lambda)}\left(\mathcal{B}^{2}-x\right)^{-1} d x,
$$

with $\gamma(\lambda)$ being a closed counterclockwise circle around the origin surrounding eigenvalues of absolute value in $[0, \lambda]$. By the analytic Fredholm theorem, the range of the projection lies in $\mathcal{D}\left(\mathcal{B}^{2}\right)$ and the projection commutes with $\mathcal{B}^{2}$. Moreover, $\Pi_{\mathcal{B}^{2},[0, \lambda]}$ is of finite rank and the 
decomposition

$$
L_{*}^{2}(M, E \oplus E)=\operatorname{Image} \Pi_{\mathcal{B}^{2},[0, \lambda]} \oplus \operatorname{Image}\left(\mathbf{1}-\Pi_{\mathcal{B}^{2},[0, \lambda]}\right),
$$

is a direct sum decomposition into closed subspaces of the Hilbert space $L_{*}^{2}(M, E \oplus E)$. Note that if $\alpha$ is unitary and hence $\mathcal{B}^{2}$ is self-adjoint, the projection $\Pi_{\mathcal{B}^{2},[0, \lambda]}$ is orthogonal. (2.6) induces a decomposition of $\widetilde{\mathcal{D}}$

$$
\widetilde{\mathcal{D}}=\widetilde{\mathcal{D}}_{[0, \lambda]} \oplus \widetilde{\mathcal{D}}_{(\lambda, \infty)} .
$$

Since $\mathbb{D}$ commutes with $\mathcal{B}, \mathcal{B}^{2}$ and hence also with $\Pi_{\mathcal{B}^{2},[0, \lambda]}$, we obtain a decomposition of $(\widetilde{\mathcal{D}}, \mathbb{D})$ into subcomplexes

$$
\begin{aligned}
& (\widetilde{\mathcal{D}}, \mathbb{D})=\left(\widetilde{\mathcal{D}}_{[0, \lambda]}, \mathbb{D}_{[0, \lambda]}\right) \oplus\left(\widetilde{\mathcal{D}}_{(\lambda, \infty)}, \mathbb{D}_{(\lambda, \infty)}\right) \\
& \text { where } \mathbb{D}_{\mathcal{I}}:=\left.\mathbb{D}\right|_{\widetilde{\mathcal{D}}_{\mathcal{I}}} \text { for } \mathcal{I}=[0, \lambda] \text { or }(\lambda, \infty) .
\end{aligned}
$$

The chirality operator $\mathbb{G}$ commutes with $\mathcal{B}, \mathcal{B}^{2}$ and respects the decomposition (2.7) so that

$$
\mathbb{G}=\mathbb{G}_{[0, \lambda]} \oplus \mathbb{G}_{(\lambda, \infty)}, \quad \mathcal{B}=\mathcal{B}^{[0, \lambda]} \oplus \mathcal{B}^{(\lambda, \infty)} .
$$

Proposition 2.10. [44, Corollary 3.14 and 3.15]. The operator $\mathcal{B}^{(\lambda, \infty)}$, $\lambda \geq 0$ is bijective. The complex $\left(\widetilde{\mathcal{D}}_{(\lambda, \infty)}, \mathbb{D}_{(\lambda, \infty)}\right)$ is acyclic and

$$
H^{*}\left(\widetilde{\mathcal{D}}_{[0, \lambda]}, \mathbb{D}_{[0, \lambda]}\right) \cong H^{*}(\widetilde{\mathcal{D}}, \mathbb{D}) \text {. }
$$

2.11. The refined torsion element. Recall the notion of a determinant lines of a finite dimensional complex $\left(C^{*}, \partial_{*}\right)$ and of its cohomology. Set

$$
\begin{aligned}
\operatorname{Det} C^{*} & =\bigotimes_{k} \operatorname{det}\left(C^{k}\right)^{(-1)^{k}}, \\
\operatorname{Det} H^{*}\left(C^{*}, \partial_{*}\right) & =\bigotimes_{k} \operatorname{det} H^{k}\left(C^{*}, \partial_{*}\right)^{(-1)^{k}},
\end{aligned}
$$

where for a vector space $V$ we denote by $\operatorname{det} V$ its top exterior power and the $(-1)$ upper index denotes the dual vector space. We follow $[5$, Section 1.1] and define the canonical isomorphism

$$
\phi: \operatorname{Det} C^{*} \rightarrow \operatorname{Det} H^{*}\left(C^{*}, \partial_{*}\right)
$$

and the refined torsion element of the complex $\left(\widetilde{\mathcal{D}}_{[0, \lambda]}, \mathbb{D}_{[0, \lambda]}\right)$

$$
\begin{aligned}
\rho_{[0, \lambda]}:= & \phi\left(c_{0} \otimes\left(c_{1}\right)^{-1} \otimes \cdots \otimes\left(c_{r}\right)^{(-1)^{r}} \otimes\left(\mathbb{G}_{[0, \lambda]} c_{r}\right)^{(-1)^{r+1}} \otimes \cdots\right. \\
& \left.\cdots \otimes\left(\mathbb{G}_{[0, \lambda]} c_{1}\right) \otimes\left(\mathbb{G}_{[0, \lambda]} c_{0}\right)^{(-1)}\right) \in \operatorname{Det}\left(H^{*}\left(\widetilde{\mathcal{D}}_{[0, \lambda]}, \mathbb{D}_{[0, \lambda]}\right)\right),
\end{aligned}
$$

where $c_{k} \in \widetilde{\mathcal{D}}_{[0, \lambda]}$ are arbitrary elements of the determinant lines, we denote the extension of $\mathbb{G}_{[0, \lambda]}$ to a mapping on determinant lines by the same letter, and for any $v \in \operatorname{det} \widetilde{\mathcal{D}}_{[0, \lambda]}$ the dual $v^{-1} \in \operatorname{det}\left(\widetilde{\mathcal{D}}_{[0, \lambda]}\right)^{-1} \equiv$ $\operatorname{det}\left(\widetilde{\mathcal{D}}_{[0, \lambda]}\right)^{*}$ is the unique element such that $v^{-1}(v)=1$. 
By Proposition 2.10 we can view $\rho_{[0, \lambda]}$ canonically as an element of $\operatorname{Det}\left(H^{*}(\widetilde{\mathcal{D}}, \mathbb{D})\right)$, which we do henceforth.

2.12. The graded determinant. The fundamental part of the construction is the graded determinant. The operator $\mathcal{B}^{(\lambda, \infty)}, \lambda \geq 0$ is bijective by Proposition 2.10 and hence by injectivity (put $\mathcal{I}=(\lambda, \infty)$ to simplify the notation)

$$
\operatorname{ker}\left(\mathbb{D}_{\mathcal{I}} \mathbb{G}_{\mathcal{I}}\right) \cap \operatorname{ker}\left(\mathbb{G}_{\mathcal{I}} \mathbb{D}_{\mathcal{I}}\right)=\{0\} .
$$

Moreover the complex $\left(\widetilde{\mathcal{D}}_{\mathcal{I}}, \mathbb{D}_{\mathcal{I}}\right)$ is acyclic by Proposition 2.10 and due to $\mathbb{G}_{\mathcal{I}}$ being an involution on $\operatorname{Im}\left(1-\Pi_{\mathcal{B}^{2},[0, \lambda]}\right)$ we have

$$
\begin{aligned}
& \operatorname{ker}\left(\mathbb{D}_{\mathcal{I}} \mathbb{G}_{\mathcal{I}}\right)=\mathbb{G}_{\mathcal{I}} \operatorname{ker}\left(\mathbb{D}_{\mathcal{I}}\right)=\mathbb{G}_{\mathcal{I}} \operatorname{Im}\left(\mathbb{D}_{\mathcal{I}}\right)=\operatorname{Im}\left(\mathbb{G}_{\mathcal{I}} \mathbb{D}_{\mathcal{I}}\right), \\
& \operatorname{ker}\left(\mathbb{G}_{\mathcal{I}} \mathbb{D}_{\mathcal{I}}\right)=\operatorname{ker}\left(\mathbb{D}_{\mathcal{I}}\right)=\operatorname{Im}\left(\mathbb{D}_{\mathcal{I}}\right)=\operatorname{Im}\left(\mathbb{D}_{\mathcal{I}} \mathbb{G}_{\mathcal{I}}\right) .
\end{aligned}
$$

We have $\operatorname{Im}\left(\mathbb{G}_{\mathcal{I}} \mathbb{D}_{\mathcal{I}}\right)+\operatorname{Im}\left(\mathbb{D}_{\mathcal{I}} \mathbb{G}_{\mathcal{I}}\right)=\operatorname{Im}\left(\mathcal{B}^{\mathcal{I}}\right)$ and by surjectivity of $\mathcal{B}^{\mathcal{I}}$ we obtain from the last three relations above

$$
\operatorname{Im}\left(1-\Pi_{\mathcal{B}^{2},[0, \lambda]}\right)=\operatorname{ker}\left(\mathbb{D}_{\mathcal{I}} \mathbb{G}_{\mathcal{I}}\right) \oplus \operatorname{ker}\left(\mathbb{G}_{\mathcal{I}} \mathbb{D}_{\mathcal{I}}\right) .
$$

Note that $\mathcal{B}$ leaves $\operatorname{ker}(\mathbb{D} \mathbb{G})$ and $\operatorname{ker}(\mathbb{G D})$ invariant. Hence, we put

$$
\begin{aligned}
& \mathcal{B}_{\text {even }}^{+,(\lambda, \infty)}:=\mathcal{B}^{(\lambda, \infty)} \uparrow \widetilde{\mathcal{D}}^{\text {even }} \cap \operatorname{ker}(\mathbb{D} \mathbb{G}), \\
& \mathcal{B}_{\text {even }}^{-,(\lambda, \infty)}:=\mathcal{B}^{(\lambda, \infty)} \uparrow \widetilde{\mathcal{D}}^{\text {even }} \cap \operatorname{ker}(\mathbb{G} \mathbb{D}) .
\end{aligned}
$$

We arrive at a direct sum decomposition

$$
\mathcal{B}_{\text {even }}^{(\lambda, \infty)}=\mathcal{B}_{\text {even }}^{+,(\lambda, \infty)} \oplus \mathcal{B}_{\text {even }}^{-,(\lambda, \infty)} .
$$

By [44], there exists an Agmon angle $\theta \in(-\pi, 0)$ for $\mathcal{B}$, which is clearly an Agmon angle for the restrictions above, as well. For strongly elliptic boundary value problems $(D, B)$ of order $\omega$ on $M$ with an Agmon angle $\theta \in(-\pi, 0)$, the associated zeta-function is defined by

$$
\zeta_{\theta}\left(s, D_{B}\right):=\sum_{\lambda \in \operatorname{Spec}\left(D_{B}\right) \backslash\{0\}} m(\lambda) \cdot \lambda_{\theta}^{-s}, \quad \operatorname{Re}(s)>\frac{\operatorname{dim} M}{\omega},
$$

where $\lambda_{\theta}^{-s}:=\exp \left(-s \cdot \log _{\theta} \lambda\right)$ and $m(\lambda)$ denotes the multiplicity of the eigenvalue $\lambda$. The zeta function is holomorphic for $\operatorname{Re}(s)>\operatorname{dim} M / \omega$ and admits a meromorphic extension to $\mathbb{C}$ with $s=0$ being a regular point. Consequently, the graded zeta-function

$$
\zeta_{g r, \theta}\left(s, \mathcal{B}_{\text {even }}^{(\lambda, \infty)}\right):=\zeta_{\theta}\left(s, \mathcal{B}_{\text {even }}^{+,(\lambda, \infty)}\right)-\zeta_{\theta}\left(s,-\mathcal{B}_{\text {even }}^{-,(\lambda, \infty)}\right), \operatorname{Re}(s) \gg 0,
$$

is regular at $s=0$ and we may introduce the following 
Definition 2.13. Let $\theta \in(-\pi, 0)$ be an Agmon angle for $\mathcal{B}^{(\lambda, \infty)}$. Then the graded determinant associated to $\mathcal{B}^{(\lambda, \infty)}$ and its Agmon angle $\theta$ is defined as follows:

$$
\operatorname{Det}_{g r, \theta}^{\prime}\left(\mathcal{B}_{\text {even }}^{(\lambda, \infty)}\right):=\exp \left(-\left.\frac{d}{d s}\right|_{s=0} \zeta_{g r, \theta}\left(s, \mathcal{B}_{\text {even }}^{(\lambda, \infty)}\right)\right) .
$$

\subsection{Refined analytic torsion.}

Proposition 2.15. [7, 44]. The element

$$
\rho(\nabla, g):=\operatorname{Det}_{g r, \theta}^{\prime}\left(\mathcal{B}_{\text {even }}^{(\lambda, \infty)}\right) \cdot \rho_{[0, \lambda]} \in \operatorname{Det}\left(H^{*}(\widetilde{\mathcal{D}}, \mathbb{D})\right)
$$

is independent of the choice of $\lambda \geq 0$ and choice of Agmon angle $\theta \in$ $(-\pi, 0)$ for the odd-signature operator $\mathcal{B}^{(\lambda, \infty)}$.

The construction of $\rho(\nabla, g)$ is in fact independent of the choice of a Hermitian metric $h^{E}$. Indeed, a variation of $h^{E}$ does not change the odd-signature operator $\mathcal{B}$ as a differential operator and different Hermitian metrics give rise to equivalent $L^{2}$-norms over compact manifolds. Hence $\mathcal{D}(\mathcal{B})$ is indeed independent of the particular choice of $h^{E}$. Independence of the choice of a Hermitian metric $h^{E}$ is essential, since for non-unitary flat vector bundles there is no canonical choice of $h^{E}$ and a Hermitian metric is fixed arbitrarily.

The refined analytic torsion is then obtained by studying the dependence of $\rho(\nabla, g)$ on the Riemannian metric. We cite the final result from [44].

Theorem 2.16. Let $(M, g)$ be an odd-dimensional oriented compact Riemannian manifold with boundary. Let $\left(E, \nabla, h^{E}\right)$ be a flat complex vector bundle over $M$. Consider the trivial vector bundle $M \times \mathbb{C}$ with a trivial connection $d$ and let $B:=\mathcal{B}(d)$ denote the associated oddsignature operator. $\eta(B)$ denotes the eta invariant of the even part $B_{\text {even. }}$ Put

$$
\widehat{\xi}(d, g):=\frac{1}{2} \sum_{k=0}^{m}(-1)^{k} \cdot k \cdot \zeta_{2 \theta}\left(s=0, B^{2} \uparrow \widetilde{\mathcal{D}}^{k}\right) .
$$

Then the refined analytic torsion of $(M, E, \nabla)$

$$
\rho_{\text {an }}(\nabla):=\rho(\nabla, g) \cdot \exp [i \pi \operatorname{rk}(E)(\eta(B)+\widehat{\xi}(d, g))]
$$

is modulo sign independent of the choice of $g$ in the interior of $M$. 


\section{Holomorphic StRucture on the Determinant Line BUNDLE}

In the next step we interpret $\rho(\alpha)$ as an analytic section of the determinant line bundle over the representation space. This requires a separate discussion of the analyticity for the refined torsion element and the graded determinant. The present section studies analyticity of the refined torsion element, while the next deals with analyticity of the graded determinant.

3.1. The determinant line bundle. The space $\mathscr{R}:=\operatorname{Rep}\left(\pi_{1}(M), \mathbb{C}^{n}\right)$ of complex $n$-dimensional representations of $\pi_{1}=\pi_{1}(M)$ has a natural structure of a complex analytic space, cf., for example, [7, §13.6] . For each $\alpha \in \operatorname{Rep}\left(\pi_{1}(M), \mathbb{C}^{n}\right)$ we denote by $E_{\alpha}$ the flat vector bundle over $M$ whose monodromy is equal to $\alpha$. Then the disjoint union

$$
\text { Det }:=\bigsqcup_{\alpha \in \mathscr{R}} \operatorname{Det}\left(H^{\bullet}\left(M, E_{\alpha}\right)\right) \otimes \operatorname{Det}\left(H^{\bullet}\left(M, \partial M, E_{\alpha}\right)\right)
$$

has a natural structure of a holomorphic line bundle over $\mathscr{R}$, called the determinant line bundle. In this section we describe this structure, using a CW-decomposition of $M$. Then, we show that the refined analytic torsion is a nowhere vanishing holomorphic section of $\mathcal{D} e t$. We continue in the notation fixed in $\S 2$.

3.2. The combinatorial cochain complex. Fix a CW-decomposition $K=\left\{e_{1}, \ldots, e_{N}\right\}$ of $M$. Let $\widetilde{K}$ denote the universal cover of $K$. Then the fundamental group $\pi_{1}(M)$ acts on $C \bullet(\widetilde{K}, \mathbb{C})$ from the right and $\mathbb{C}^{n}$ is a left module over the group ring $\mathbb{C}\left[\pi_{1}\right]$ via the representation $\alpha$. Then the cochain complex $C^{\bullet}(K, \alpha)$ is defined as

$$
C^{\bullet}(K, \alpha):=C^{\bullet}(\widetilde{K}, \mathbb{C}) \otimes_{\mathbb{C}\left[\pi_{1}\right]} \mathbb{C}^{n}
$$

For each cell $e_{j}$, fix a lift $\widetilde{e}_{j}$, a cell of the CW-decomposition of $\widetilde{M}$, such that $\pi\left(\widetilde{e}_{j}\right)=e_{j}$. By definition, the pull-back of the bundle $E_{\alpha}$ to $\widetilde{M}$ is the trivial bundle $\widetilde{M} \times \mathbb{C}^{n} \rightarrow \widetilde{M}$. Hence, the choice of the cells $\widetilde{e}_{1}, \ldots, \widetilde{e}_{N}$ identifies the cochain complex $C^{\bullet}(K, \alpha)$ of the CW-complex $K$ with coefficients in $E_{\alpha}$ with the complex

$$
0 \rightarrow \mathbb{C}^{n \cdot k_{0}} \stackrel{\partial_{0}(\alpha)}{\longrightarrow} \mathbb{C}^{n \cdot k_{1}} \stackrel{\partial_{1}(\alpha)}{\longrightarrow} \cdots \stackrel{\partial_{m-1}(\alpha)}{\longrightarrow} \mathbb{C}^{n \cdot k_{m}} \rightarrow 0
$$

where $k_{j} \in \mathbb{Z}_{\geq 0}(j=0, \ldots, m=\operatorname{dim} M)$ is equal to the number of $j$-dimensional cells of $K$ and the differentials $\partial_{j}(\alpha)$ are $\left(n k_{j} \times n k_{j-1}\right)$ matrices depending analytically on $\alpha \in \operatorname{Rep}\left(\pi_{1}(M), \mathbb{C}^{n}\right)$. 
The cohomology of the complex (3.3) is canonically isomorphic to $H^{\bullet}\left(M, E_{\alpha}\right)$. Let

$$
\phi_{C \cdot(K, \alpha)}: \operatorname{Det}\left(C^{\bullet}(K, \alpha)\right) \longrightarrow \operatorname{Det}\left(H^{\bullet}\left(M, E_{\alpha}\right)\right)
$$

denote the canonical isomorphism, cf. formula (2.13) of [5] .

3.3. A non-zero element of Det $\left(H^{\bullet}\left(M, E_{\alpha}\right)\right)$. The standard bases of $\mathbb{C}^{n \cdot k_{j}}(j=0, \ldots, m)$ define an element $c \in \operatorname{Det}\left(C^{\bullet}(K, \alpha)\right)$, and, hence, an isomorphism $\psi_{\alpha}: \mathbb{C} \longrightarrow \operatorname{Det}\left(C^{\bullet}(K, \alpha)\right)$ with $\psi_{\alpha}(z)=z \cdot c$. Then for each $\alpha \in \operatorname{Rep}\left(\pi_{1}(M), \mathbb{C}^{n}\right)$ we define

$$
\sigma(\alpha)=\phi_{C \cdot(K, \alpha)}\left(\psi_{\alpha}(1)\right) \in \operatorname{Det}\left(H^{\bullet}\left(M, E_{\alpha}\right)\right),
$$

a non-zero element of $\operatorname{Det}\left(H^{\bullet}\left(M, E_{\alpha}\right)\right)$. Of course, this element depends on the choice of the lifts $\widetilde{e}_{1}, \ldots, \widetilde{e}_{N}$.

3.4. A non-zero element of $\operatorname{Det}\left(H^{\bullet}\left(M, \partial M, E_{\alpha}\right)\right)$. Let now $K^{\prime}$ denote the CW-decomposition of $\partial M$ induced by $K$. Then $K^{\prime} \subset K$ and the choice of the lifts $\widetilde{e}_{i}$ made above identify the cochain complex $C^{\bullet}\left(K^{\prime}, \alpha\right)$ of the CW-complex $K^{\prime}$ with coefficients in $E_{\alpha}$ with the complex

$$
0 \rightarrow \mathbb{C}^{n \cdot l_{0}} \stackrel{\partial_{0}(\alpha)}{\longrightarrow} \mathbb{C}^{n \cdot l_{1}} \stackrel{\partial_{1}(\alpha)}{\longrightarrow} \cdots \stackrel{\partial_{m-1}(\alpha)}{\longrightarrow} \mathbb{C}^{n \cdot l_{m-1}} \rightarrow 0,
$$

As above, the standard bases of $\mathbb{C}^{n \cdot l_{j}}(j=1, \ldots, m-1)$ defines a canonical element of $\operatorname{Det}\left(C^{\bullet}\left(K^{\prime}, \alpha\right)\right)$ and an isomorphism $\psi_{\alpha}^{\prime}$ from $\mathbb{C}$ onto $\operatorname{Det}\left(C^{\bullet}(K, \alpha)\right)$. Thus we define

$$
\sigma^{\prime}(\alpha)=\phi_{C \cdot\left(K^{\prime}, \alpha\right)}\left(\psi_{\alpha}^{\prime}(1)\right) \in \operatorname{Det}\left(H^{\bullet}\left(\partial M, E_{\alpha}\right)\right),
$$

where $\alpha^{\prime}$ is the restriction of the representation $\alpha$ to $\pi_{1}(\partial M)$ and $E_{\alpha}^{\prime}$ is the restriction of $E_{\alpha}$ to $\partial M$. Consider the quotient complex

$$
C^{\bullet}\left(K, K^{\prime}, \alpha\right):=C^{\bullet}(K, \alpha) / C^{\bullet}\left(K^{\prime}, \alpha\right) .
$$

Using (3.3) and (3.6) we can identify $\operatorname{Det}\left(C^{\bullet}\left(K, K^{\prime}, \alpha\right)\right)$ with $\mathbb{C}$ thus constructing a map

$$
\psi_{\alpha}^{\prime \prime}: \mathbb{C} \longrightarrow \operatorname{Det}\left(C^{\bullet}\left(K, K^{\prime}, \alpha\right)\right) .
$$

The cohomology of the complex $C^{\bullet}\left(K, K^{\prime}, \alpha\right)$ is canonically isomorphic to the relative cohomology $H^{\bullet}\left(M, \partial M, E_{\alpha}\right)$. For each $\alpha$ we define a non-zero element of $\operatorname{Det}\left(H^{\bullet}\left(M, \partial M, E_{\alpha}\right)\right)$ by the formula

$$
\sigma^{\prime \prime}(\alpha)=\phi_{C} \cdot\left(K, K^{\prime}, \alpha\right)\left(\psi_{\alpha}^{\prime \prime}(1)\right) \in \operatorname{Det}\left(H^{\bullet}\left(M, \partial M, E_{\alpha}\right)\right),
$$


3.5. The holomorphic structure on Det. Recall that the elements $\sigma(\alpha)$ and $\sigma^{\prime \prime}(\alpha)$ are defined in (3.5) and (3.9). Consider the map

$$
\begin{aligned}
\tau: \alpha & \mapsto \sigma(\alpha) \otimes \sigma^{\prime \prime}(\alpha) \\
& \in \operatorname{Det}\left(H^{\bullet}\left(M, E_{\alpha}\right)\right) \otimes \operatorname{Det}\left(H^{\bullet}\left(M, \partial M, E_{\alpha}\right)\right),
\end{aligned}
$$

where $\alpha \in \operatorname{Rep}\left(\pi_{1}(M), \mathbb{C}^{n}\right)$, is a nowhere vanishing section of the determinant line bundle $\mathcal{D}$ et over $\operatorname{Rep}\left(\pi_{1}(M), \mathbb{C}^{n}\right)$.

Definition 3.6. We say that a section $s(\alpha)$ of Det is holomorphic if there exists a holomorphic function $f(\alpha)$ on $\operatorname{Rep}\left(\pi_{1}(M), \mathbb{C}^{n}\right)$, such that $s(\alpha)=f(\alpha) \cdot \tau(\alpha)$.

This defines a holomorphic structure on Det, which is independent of the choice of the lifts $\widetilde{e}_{1}, \ldots, \widetilde{e}_{N}$ of $e_{1}, \ldots, e_{N}$, since for a different choice of lifts the section $\tau(\alpha)$ will be multiplied by a constant. In the next subsection we show that this holomorphic structure is also independent of the CW-decomposition $K$ of $M$.

3.7. The Farber-Turaev torsion. The choice of the lifts $\widetilde{e}_{1}, \ldots, \widetilde{e}_{N}$ of $e_{1}, \ldots, e_{N}$ determines an Euler structure $\varepsilon$ on $M$, while the ordering of the cells $e_{1}, \ldots, e_{N}$ determines a cohomological orientation $\mathfrak{o}$, cf. [43, §20]. Moreover, every Euler structure and every cohomological orientation can be obtained in this way. The Farber-Turaev torsion $\rho_{\varepsilon, \mathfrak{o}}(\alpha)$, corresponding to the pair $(\varepsilon, \mathfrak{o})$, is, by definition, $[21, \S 6]$, equal to the element $\sigma(\alpha)$ defined in (3.5). Since the Farber-Turaev torsion is independent of the choice of the $\mathrm{CW}$-decomposition of $M$, cf. $[42,21]$, we conclude that the element $\sigma(\alpha)$ is also independent of the CW-decomposition, but only depends on the Euler structure and the cohomological orientation.

The lifts $\widetilde{e}_{j}$ and the ordering of the cells also defines an Euler structure $\varepsilon^{\prime}$ and a cohomological orientation $\mathfrak{o}^{\prime}$ of $\partial M$. The element $\sigma^{\prime}(\alpha)$ defined in (3.7) is equal to the Farber-Turaev torsion $\rho_{\varepsilon^{\prime}, \mathfrak{o}^{\prime}}\left(\alpha^{\prime}\right)$ where $\alpha^{\prime}$ is the restriction of the representation $\alpha$ to $\pi_{1}(\partial M)$. Let

$$
\mu: \operatorname{Det}\left(C^{\bullet}\left(K^{\prime}, \alpha\right)\right) \otimes \operatorname{Det}\left(C^{\bullet}\left(K, K^{\prime}, \alpha\right)\right) \longrightarrow \operatorname{Det}\left(C^{\bullet}(K, \alpha)\right)
$$

denote the fusion isomorphism, cf. [5, §2.6] . Using this isomorphism we define the map

$$
\begin{gathered}
\nu: \operatorname{Det}\left(H^{\bullet}\left(\partial M, E_{\alpha}\right)\right) \otimes \operatorname{Det}\left(H^{\bullet}\left(M, \partial M, E_{\alpha}\right)\right) \longrightarrow \operatorname{Det}\left(H^{\bullet}\left(M, E_{\alpha}\right)\right), \\
\nu:=\phi_{C}(K, \alpha) \circ \mu \circ\left(\phi_{C^{\bullet}\left(K^{\prime}, \alpha\right)}^{-1} \otimes \phi_{C^{\bullet}\left(K, K^{\prime}, \alpha\right)}^{-1}\right) .
\end{gathered}
$$


It follows from the construction that the elements $\sigma(\alpha), \sigma^{\prime}(\alpha)$ and $\sigma^{\prime \prime}(\alpha)$ defined in (3.11), (3.5), (3.7), and (3.9) satisfy the equality

$$
\nu\left(\sigma^{\prime}(\alpha) \otimes \sigma^{\prime \prime}(\alpha)\right)= \pm \sigma(\alpha),
$$

where the sign depends only on the dimensions of the spaces $C^{\bullet}(M, \alpha)$ and $C^{\bullet}(\partial M, \alpha)$ but not on the representation $\alpha$. Since $\sigma(\alpha)$ and $\sigma^{\prime}(\alpha)$ are independent of the CW-decomposition, it follows that $\sigma^{\prime \prime}(\alpha)$ is independent of CW-decompositon up to a sign. In fact $\sigma^{\prime \prime}(\alpha)$ can be considered as the definition of the relative Farber-Turaev torsion. It follows that the section $\tau(\alpha)$ defined in (3.10) is also an independent of the CW-decomposition up to a sign. Hence so is the holomorphic structure defined in Definition 3.6.

3.8. The acyclic case. Let $\operatorname{Rep}_{0}\left(\pi_{1}(M), \mathbb{C}^{n}\right) \subset \operatorname{Rep}\left(\pi_{1}(M), \mathbb{C}^{n}\right)$ denote the set of representations such that $H^{\bullet}\left(M, E_{\alpha}\right)=0, H^{\bullet}\left(\partial M, E_{\alpha^{\prime}}\right)=$ 0 , and $H^{\bullet}\left(M, \partial M, E_{\alpha}\right)=0$. Then the determinant lines $\operatorname{Det}\left(H^{\bullet}\left(M, E_{\alpha}\right)\right)$, $\operatorname{Det}\left(H^{\bullet}\left(M, \partial M, E_{\alpha^{\prime}}\right)\right)$, and $\operatorname{Det}\left(H^{\bullet}\left(M, \partial M . E_{\alpha}\right)\right)$ are canonically isomorphic to $\mathbb{C}$. Hence, the Farber-Turaev torsions $\rho_{\varepsilon, \mathfrak{o}}(\alpha)$ and $\rho_{\varepsilon^{\prime}, \mathfrak{o}^{\prime}}(\alpha)$ can be viewed as a complex-valued functions on $\operatorname{Rep}_{0}\left(\pi_{1}(M), \mathbb{C}^{n}\right)$. It is easy to see, cf. [14, Theorem 4.3], that these functions are holomorphic on $\operatorname{Rep}_{0}\left(\pi_{1}(M), \mathbb{C}^{n}\right)$. Moreover, they are rational functions on $\operatorname{Rep}\left(\pi_{1}(M), \mathbb{C}^{n}\right)$, all whose poles are in

$$
\operatorname{Rep}\left(\pi_{1}(M), \mathbb{C}^{n}\right) \backslash \operatorname{Rep}_{0}\left(\pi_{1}(M), \mathbb{C}^{n}\right) .
$$

In particular, the holomorphic structure on Det, which we defined above, coincides, when restricted to $\operatorname{Rep}_{0}\left(\pi_{1}(M), \mathbb{C}^{n}\right)$, with the natural holomorphic structure obtained from the canonical isomorphism

$$
\left.\operatorname{Det}\right|_{\operatorname{Rep}_{0}\left(\pi_{1}(M), \mathbb{C}^{n}\right)} \simeq \operatorname{Rep}_{0}\left(\pi_{1}(M), \mathbb{C}^{n}\right) \times \mathbb{C} .
$$

\section{The GRADED Determinant AS A HOLOMORPHIC FUnCTION}

Fix $\alpha_{0} \in \operatorname{Rep}\left(\pi_{1}(M), \mathbb{C}^{n}\right)$. Fix a number $\lambda \geq 0$ which is not in the spectrum of the square $\mathcal{B}_{\alpha_{0}}^{2}$ of the odd signature operator $\mathcal{B}_{\alpha_{0}}=$ $\mathcal{B}\left(\nabla_{\alpha_{0}}, g\right)$. Then there is a neighborhood $U_{\lambda} \subset \operatorname{Rep}\left(\pi_{1}(M), \mathbb{C}^{n}\right)$ of $\alpha_{0}$ such that $\lambda$ is not an eigenvalue of $\mathcal{B}_{\alpha}^{2}$ for all $\alpha \in U_{\lambda}$. Denote by $\mathcal{B}_{\alpha}^{(\lambda, \infty)}$ the restriction of $\mathcal{B}_{\alpha}$ to the spectral subspace of $\mathcal{B}_{\alpha}^{2}$ corresponding to the spectral set $(\lambda, \infty)$. Then $\mathcal{B}_{\alpha}^{(\lambda, \infty)}$ is an invertible operator. Let $\theta \in(-\pi / 2,0)$ be an Agmon angle for $\mathcal{B}_{\alpha_{0}}^{(0, \lambda)}$ and assume that there are no eigenvalues of $\mathcal{B}_{\alpha_{0}}^{(\lambda, \infty)}$ in the solid angles $L_{(-\pi / 2, \lambda]}$ and $L_{(\pi / 2, \lambda+\pi / 2]}$. Then there exists a neighborhood $U_{\lambda, \theta} \subset U_{\lambda}$ of $\alpha_{0}$ such that $\theta$ is also an Agmon angle for $B_{\alpha}^{(\lambda, \infty)}$ for all $\alpha \in U_{\lambda, \theta}$. In this section we prove 
that the graded determinant $\operatorname{Det}_{\mathrm{gr}, \theta}^{\prime}\left(\mathcal{B}_{\alpha, \text { even }}^{(\lambda, \infty)}\right)$ is a holomorphic function on $U_{\lambda, \theta}$. Our main result in this section is the following

Theorem 4.1. Let $\mathcal{O} \subset \mathbb{C}$ be a connected open neighborhood of 0 . Let

$$
\gamma: \mathcal{O} \rightarrow U_{\lambda, \theta} \subset \operatorname{Rep}\left(\pi_{1}(M), \mathbb{C}^{n}\right)
$$

be a holomorphic curve such that $\gamma(0)=\alpha_{0}$. Then the function

$$
z \mapsto \operatorname{Det}_{\mathrm{gr}, \theta}^{\prime}\left(\mathcal{B}_{\gamma(z), \text { even }}^{(\lambda, \infty)}\right)
$$

is holomorphic in a neighborhood of 0 .

An immediate consequence is the following

Corollary 4.2. Suppose $V \subset U_{\lambda, \theta}$ is an open subset such that all points $\alpha \in V$ are regular points of the complex algebraic set $\operatorname{Rep}\left(\pi_{1}(M), \mathbb{C}^{n}\right)$. Then the map

$$
\text { Det }: V \longrightarrow \mathbb{C}, \quad \text { Det }: \alpha \mapsto \operatorname{Det}(\alpha):=\operatorname{Det}_{\mathrm{gr}, \theta}^{\prime}\left(\mathcal{B}_{\alpha, \text { even }}^{(\lambda, \infty)}\right) .
$$

is holomorphic.

Proof. By Hartogs' theorem (cf., for example, [27, Th. 2.2.8]), a function on a smooth algebraic variety is holomorphic if its restriction to each holomorphic curve is holomorphic. Hence, the corollary follows immediately from Theorem 4.1.

The rest of this section is occupied with the proof of Theorem 4.1.

4.3. A germ of connections. Let us introduce some additional notations. Let $E$ be a vector bundle over $M$ and let $\mathcal{C}(E)$ denote the affine space of (not necessarily flat) connections on $E$. We endow $\mathcal{C}(E)$ with the the Fréchet topology on $\mathcal{C}(E)$ introduced in Section 13.1 of [7] .

Fix a base point $x_{*} \in M$ and let $E_{x_{*}}$ denote the fiber of $E$ over $x_{*}$. We will identify $E_{x_{*}}$ with $\mathbb{C}^{n}$ and $\pi_{1}\left(M, x_{*}\right)$ with $\pi_{1}(M)$.

For $\nabla \in \mathcal{C}(E)$ and a closed path $\phi:[0,1] \rightarrow M$ with $\phi(0)=\phi(1)=$ $x_{*}$, we denote by $\operatorname{Mon}_{\nabla}(\phi) \in$ End $E_{x_{*}} \simeq \operatorname{Mat}_{n \times n}(\mathbb{C})$ the monodromy of $\nabla$ along $\phi$. Note that, if $\nabla$ is flat then $\operatorname{Mon}_{\nabla}(\phi)$ depends only on the class $[\phi]$ of $\phi$ in $\pi_{1}(M)$. Hence, if $\nabla$ is flat, then the map $\phi \mapsto$ $\operatorname{Mon}_{\nabla}(\phi)$ defines an element of $\operatorname{Rep}\left(\pi_{1}(M), \mathbb{C}^{n}\right)$, called the monodromy representation of $\nabla$.

Suppose now that $\mathcal{O} \subset \mathbb{C}$ is a connected open neighborhood of 0 . For simplicity we also assume that $\mathcal{O}$ is convex. Let

$$
\gamma: \mathcal{O} \rightarrow \operatorname{Rep}\left(\pi_{1}(M), \mathbb{C}^{n}\right)
$$

be a holomorphic curve with $\gamma(0)=\alpha_{0}$. The operator $\mathcal{B}_{\gamma(z)}$ is constructed using a flat connection $\nabla_{\gamma(z)}$ whose monodromy is equal to 
$\gamma(z)$. Unfortunately, there is no a canonical choice of such connection. Though the graded determinant $\operatorname{Det}_{\mathrm{gr}, \theta}^{\prime}\left(\mathcal{B}_{\gamma(z) \text {,even }}^{(\lambda, \infty)}\right)$ is independent of this choice, to study the dependence of this determinant on $z \in \mathcal{O}$ we need to choose a family of connections $\nabla_{\gamma(z)}$. The main difficulty in the proof of Theorem 4.1 is that it is not clear whether there exists a holomorphic family $\nabla_{\gamma(z)}$ with $\operatorname{Mon}_{\nabla_{\gamma(z)}}=\gamma(z)$. We shall now explain how to circumvent this difficulty.

By Proposition 4.5 of [23], all the bundles $E_{\gamma(z)}, z \in \mathcal{O}$, are isomorphic to each other. Moreover, we have the following lemma.

Lemma 4.4. There exists a vector bundle $E \rightarrow M$ and a real differentiable family of flat connections $\nabla_{\gamma(z)}, z \in \mathcal{O}$, on $E$, such that the monodromy representation of $\nabla_{\gamma(z)}$ is equal to $\gamma(z)$ for all $z \in \mathcal{O}$.

Proof. By Lemma 3.3 of [1] there exists a smooth vector bundle

$$
\tilde{E} \rightarrow M \times \mathcal{O}
$$

and a smooth connection $\tilde{\nabla}$ on $\tilde{E}$, whose restriction $\tilde{\nabla}_{z}$ to

$$
\left.\tilde{E}\right|_{M \times\{z\}} \rightarrow M \times\{z\}
$$

is flat for each $z \in \mathcal{O}$, and such that the monodromy of $\tilde{\nabla}_{z}$ is equal to $\gamma(z)$.

Set $E:=\left.\tilde{E}\right|_{M \times\{0\}}$ and let

$$
\Phi_{z}:\left.E \rightarrow \tilde{E}\right|_{M \times\{z\}}
$$

denote the parallel transport along the intervals

$$
\{(m, t z): m \in M, t \in[0,1]\} \subset M \times \mathcal{O} .
$$

Then

$$
\nabla_{\gamma(z)}:=\Phi_{z}^{-1} \circ \tilde{\nabla}_{z} \circ \Phi_{z}, \quad z \in \mathcal{O}
$$

is a smooth family of connections on $E$, and the monodromy of $\nabla_{\gamma(z)}$ is equal to $\gamma(z)$.

Furthermore, Lemma B.6 of [7] shows that the family $\nabla_{\gamma(z)}$ can be chosen so that there exists a one-form $\omega \in \Omega^{1}(M$, End $E)$ such that

$$
\nabla_{\gamma(z)}=\nabla_{\alpha_{0}}+z \cdot \omega+o(z)
$$

where $o(z)$ is understood in the sense of the Fréchet topology on $\mathcal{C}(E)$ introduced in Section 13.1 of [7], and always refers to the behavior as $z \rightarrow 0$. 
Remark 4.5. Lemma 4.4 asserts that the family $\nabla_{\gamma(z)}$ can be chosen to be real differentiable at every point $z \in \mathcal{O}$. We don't know whether it can be chosen to be complex differentiable on the whole set $\mathcal{O}$. However, the equation (4.2) implies that it can be chosen to be complex differentiable at 0 .

4.6. The family of projections. Let $\operatorname{Flat}(E) \subset \mathcal{C}(E)$ denote the set of flat connections on $E$ and consider a curve $\nabla(z) \in \operatorname{Flat}(E), z \in \mathcal{O}$, of connections such that $\operatorname{Mon} \nabla(0)=\alpha_{0}$. We will assume that it is complex differentiable at 0 in the sense that

$$
\nabla(z)=\nabla_{\alpha_{0}}+z \cdot \omega+o(z), \quad z \rightarrow 0,
$$

where $\omega \in \Omega^{1}(M$, End $E)$. We denote by

$$
\mathbb{D}(z):=\nabla(z)_{\min } \oplus \nabla(z)_{\max }
$$

and by $\mathcal{B}(z)$ the corresponding odd signature operator the sense of $\S 2$. Let $P(z)=\Pi_{\mathcal{B}^{2}(z),(\lambda, \infty)}$ denote the spectral projection of the operator $\mathcal{B}(z)^{2}$ onto the subspace $\widetilde{\mathcal{D}}_{(\lambda, \infty)}$ spanned by eigenforms of $\mathcal{B}(z)^{2}$ with eigenvalues in $(\lambda, \infty)$.

From (2.12) we conclude that the space $L_{*}^{2}(M, E \oplus E)$ of $L^{2}$-differential forms is a direct sum

$$
L_{*}^{2}(M, E \oplus E)=\operatorname{Im}(I-P(z)) \oplus \overline{\operatorname{Im}(P(z) \mathbb{D}(z))} \oplus \overline{\operatorname{Im}(P(z) \mathbb{G D}(z))} .
$$

Consider the corresponding orthogonal projections

$$
\begin{aligned}
P_{+}(z): L_{*}^{2}(M, E \oplus E) & \longrightarrow \overline{\operatorname{Im}(P(z) \mathbb{D}(z))}, \\
P_{-}(z): L_{*}^{2}(M, E \oplus E) & \longrightarrow \overline{\operatorname{Im}(P(z) \mathbb{G D}(z))} .
\end{aligned}
$$

Then

$$
\operatorname{Im} P(z)=\operatorname{Im} P_{+}(z) \oplus \operatorname{Im} P_{-}(z) .
$$

Lemma 4.7. There exist bounded operators $A_{ \pm}$on $L_{*}^{2}(M, E \oplus E)$ with

$$
\begin{aligned}
P_{ \pm}(z)=P_{ \pm}(0) & +z\left(P_{ \pm}(0) A_{ \pm}\left(\mathrm{Id}-P_{ \pm}(0)\right)\right. \\
& \left.+\left(\operatorname{Id}-P_{ \pm}(0)\right) A_{ \pm} P_{ \pm}(0)\right)+o(z) .
\end{aligned}
$$

Proof. For small enough $z \in \mathcal{O}$ the operators $P_{ \pm}(z)$ depend smoothly on $z$, there exist bounded operators $A_{ \pm}$and $A$ such that

$$
\begin{aligned}
P_{ \pm}(z) & =P_{ \pm}(0)+z A_{ \pm}+o(z), \\
P(z) & =P(0)+z A+o(z) .
\end{aligned}
$$


Using the decomposition

$$
\begin{aligned}
A= & P_{ \pm}(0) A P_{ \pm}(0)+\left(\mathrm{Id}-P_{ \pm}(0)\right) A P_{ \pm}(0) \\
& +P_{ \pm}(0) A\left(\mathrm{Id}-P_{ \pm}(0)\right)+\left(\mathrm{Id}-P_{ \pm}(0)\right) A\left(\mathrm{Id}-P_{ \pm}(0)\right)
\end{aligned}
$$

and the equality $P_{ \pm}(z)^{2}=P_{ \pm}(z)$ we obtain

$$
\begin{aligned}
& P_{ \pm}(z)=P_{ \pm}(z)^{2}=P_{ \pm}(0)+2 z P_{ \pm}(0) A P_{ \pm}(0) \\
& \quad+z\left(\operatorname{Id}-P_{ \pm}(0)\right) A P_{ \pm}(0)+z P_{ \pm}(0) A\left(\mathrm{Id}-P_{ \pm}(0)\right)+o(z) .
\end{aligned}
$$

Comparing (4.6) and (4.8) and using (4.7) we conclude that

$$
P_{ \pm}(0) A P_{ \pm}(0)=\left(\operatorname{Id}-P_{ \pm}(0)\right) A\left(\operatorname{Id}-P_{ \pm}(0)\right)=0 .
$$

The equality (4.5) follows now from (4.6) and (4.7).

4.8. The partial derivatives of the graded determinant. In terms of the projections $P_{ \pm}(z)$ introduced in the previous section the odd signature operator $\mathcal{B}^{(\lambda, \infty)}$ can be written in the form

$$
\begin{aligned}
\mathcal{B}^{(\lambda, \infty)}(z) & =(\mathbb{D}(z) \mathbb{G}+\mathbb{G D}(z)) P(z) \\
= & \mathbb{D}(z) \mathbb{G} P_{+}(z)+\mathbb{G} \mathbb{D}(z) P_{-}(z) .
\end{aligned}
$$

Hence, we may write

$$
\operatorname{Det}_{\text {gr }, \theta}^{\prime}\left(\mathcal{B}_{\text {even }}^{(\lambda, \infty)}(z)\right)=\frac{\operatorname{Det}_{\theta}^{\prime}\left(\mathbb{D}(z) \mathbb{G} P_{+}(z) \uparrow L_{\text {even }}^{2}(M, E \oplus E)\right)}{\operatorname{Det}_{\theta}^{\prime}\left(-\mathbb{G D}(z) P_{-}(z)\left\lceil L_{\text {even }}^{2}(M, E \oplus E)\right)\right.}
$$

Consider a curve $\kappa:(-1,1) \rightarrow \mathcal{O}$ such that $\kappa(0)=\alpha_{0}$. To simplify the notation, set

$$
\begin{aligned}
& D_{+}(z):=\mathbb{D}(z) \mathbb{G} P_{+}(z) \uparrow L_{\text {even }}^{2}(M, E \oplus E), \\
& D_{-}(z):=-\mathbb{G} \mathbb{D}(z) P_{-}(z) \uparrow L_{\text {even }}^{2}(M, E \oplus E),
\end{aligned}
$$

and also

$\mathbb{D}^{\prime}:=\left.\frac{d}{d t} \mathbb{D}(\kappa(t))\right|_{t=0}, P_{ \pm}^{\prime}:=\left.\frac{d}{d t} P_{ \pm}(\kappa(t))\right|_{t=0}, D_{ \pm}^{\prime}=\left.\frac{d}{d t} D_{ \pm}(\kappa(t))\right|_{t=0}$

With this notation we have

$$
\operatorname{Det}_{\mathrm{gr}, \theta}^{\prime}\left(\mathcal{B}_{\text {even }}^{(\lambda, \infty)}(z)\right)=\frac{\operatorname{Det}_{\theta}^{\prime}\left(D_{+}(z)\right)}{\operatorname{Det}_{\theta}^{\prime}\left(D_{-}(z)\right)} .
$$

We consider the function

$$
F(z)=\frac{\operatorname{Det}_{\theta}^{\prime}\left(\mathbb{D}(z) \mathbb{G} P_{+}\left(\alpha_{0}\right) \uparrow L_{\text {even }}^{2}(M, E \oplus E)\right)}{\operatorname{Det}_{\theta}^{\prime}\left(-\mathbb{G} \mathbb{D}(z) P_{-}\left(\alpha_{0}\right) \uparrow L_{\text {even }}^{2}(M, E \oplus E)\right)} .
$$

Notice that the right hand side of (4.13) is similar to the right hand side of (4.12) but $P_{ \pm}(z)$ is replaced by $P_{ \pm}(0)=P_{ \pm}\left(\alpha_{0}\right)$. In particular,

$$
F(0)=\operatorname{Det}_{\mathrm{gr}, \theta}^{\prime}\left(\mathcal{B}_{\text {even }}^{(\lambda, \infty)}(0)\right) \text {. }
$$


Lemma 4.9. Then for any curve $\kappa:(-1,1) \rightarrow \mathcal{O}$ with $\kappa(0)=\alpha_{0}$ we have

$$
\left.\frac{d}{d t}\right|_{t=0} \log \operatorname{Det}_{\mathrm{gr}, \theta}^{\prime}\left(\mathcal{B}_{\mathrm{even}}^{(\lambda, \infty)}(\kappa(t))\right)=\left.\frac{d}{d t}\right|_{t=0} \log F(\kappa(t)) .
$$

Proof. Using Lemma 4.7 and the equality

$$
\mathbb{D}\left(\alpha_{0}\right) \mathbb{G} P_{+}\left(\alpha_{0}\right)=P_{+}\left(\alpha_{0}\right) \mathbb{D}\left(\alpha_{0}\right) \mathbb{G},
$$

we obtain

$$
\begin{aligned}
D_{+}^{\prime}=\mathbb{D}^{\prime} \mathbb{G} P_{+}\left(\alpha_{0}\right) & +P_{+}\left(\alpha_{0}\right) \mathbb{D}\left(\alpha_{0}\right) \mathbb{G} A_{+}\left(\mathrm{Id}-P_{+}\left(\alpha_{0}\right)\right) \\
& +\left(\operatorname{Id}-P_{+}\left(\alpha_{0}\right)\right) \mathbb{D}\left(\alpha_{0}\right) \mathbb{G} A_{+} P_{+}\left(\alpha_{0}\right) .
\end{aligned}
$$

By the variation formula for the logarithm of the determinant, cf., for example, Section 3.7 of [13], we have

$$
\begin{aligned}
& \left.\frac{d}{d t}\right|_{t=0} \log \operatorname{Det}_{\theta}^{\prime} D_{+}(\kappa(t))=\left.\operatorname{Tr} D_{+}^{-s-1}\left(\alpha_{0}\right) D_{+}^{\prime}\right|_{s=0} \\
& =\left.\operatorname{Tr} D_{+}^{-s-1}\left(\alpha_{0}\right) \mathbb{D}^{\prime} \mathbb{G} P_{+}(0)\right|_{s=0}+\left.\operatorname{Tr} D_{+}^{-s-1}\left(\alpha_{0}\right) \mathbb{D}_{\alpha_{0}} \mathbb{G} P_{+}^{\prime}\right|_{s=0} .
\end{aligned}
$$

Using (4.5) and the fact that the operators $D_{+}(0)$ and $\mathbb{D}_{\alpha_{0}} \mathbb{G}$ commute with $P_{+}(0)$ we conclude that

$$
\begin{gathered}
\operatorname{Tr} D_{+}^{-s-1}(0) \mathbb{D}_{\alpha_{0}} \mathbb{G} P_{+}^{\prime}=\operatorname{Tr}\left(\operatorname{Id}-P_{+}(0)\right) D_{+}^{-s-1}(0) \mathbb{D}_{\alpha_{0}} \mathbb{G} A_{+} P_{+}(0) \\
+\operatorname{Tr} P_{+}(0) D_{+}^{-s-1}(0) \mathbb{D}_{\alpha_{0}} \mathbb{G} A_{+}\left(\mathrm{Id}-P_{+}(0)\right)=0 .
\end{gathered}
$$

Combining (4.17) and (4.18) we conclude that

$$
\begin{aligned}
& \left.\frac{d}{d t}\right|_{t=0} \log \operatorname{Det}_{\theta}^{\prime} D_{+}(\kappa(t))=\left.\operatorname{Tr} D_{+}^{-s-1}\left(\alpha_{0}\right) \mathbb{D}^{\prime} \mathbb{G} P_{+}(0)\right|_{s=0} \\
= & \left.\frac{d}{d t}\right|_{t=0} \operatorname{Det}_{\theta}^{\prime}\left(\mathbb{D}(\kappa(t)) \mathbb{G} P_{+}\left(\alpha_{0}\right) \uparrow L_{\text {even }}^{2}(M, E \oplus E)\right) .
\end{aligned}
$$

Similarly,

$$
\begin{aligned}
& \left.\frac{d}{d t}\right|_{t=0} \log \operatorname{Det}_{\theta}^{\prime} D_{-}(\kappa(t)) \\
= & \left.\frac{d}{d t}\right|_{t=0} \log \operatorname{Det}_{\theta}^{\prime}\left(-\mathbb{G D}(\kappa(t)) P_{-}\left(\alpha_{0}\right) \uparrow L_{\text {even }}^{2}(M, E \oplus E)\right) .
\end{aligned}
$$

From (4.12), (4.13), (4.19) and (4.20) we obtain (4.14).

Proposition 4.10. Let $\nabla(z)(z \in \mathcal{O})$ be a family of flat connections such that as $z \rightarrow 0$

$$
\nabla(z)=\nabla_{\alpha_{0}}+z \cdot \omega+o(z) .
$$

Then the function

$$
z \mapsto f(z):=\operatorname{Det}_{\mathrm{gr}, \theta}^{\prime}\left(\mathcal{B}_{\text {even }}^{(\lambda, \infty)}\left(\nabla_{\gamma(z)}, g\right)\right)
$$


is complex differentiable at zero. In other words, there exists a complex number $A$ such that

$$
\begin{aligned}
\operatorname{Det}_{\mathrm{gr}, \theta}^{\prime}\left(\mathcal{B}_{\text {even }}^{(\lambda, \infty)}\left(\nabla_{\gamma(z)}, g\right)\right) & =\operatorname{Det}_{\mathrm{gr}, \theta}^{\prime}\left(\mathcal{B}_{\text {even }}^{(\lambda, \infty)}\left(\nabla_{\gamma(0)}, g\right)\right) \\
& +A \cdot z+o(z) .
\end{aligned}
$$

Proof. By (4.14) it is enough to show that

$$
F(z)=F(0)+A \cdot z+o(z) .
$$

Set $z=x+i y$. Using the variation formula for the logarithm of the determinant as in the proof of Lemma 4.9, one easily sees that

$$
i \frac{\partial}{\partial x} \log F(z)=\frac{\partial}{\partial y} \log F(z)
$$

which is equivalent to (4.24).

4.11. Proof of Theorem 4.1. It follows from Proposition 4.10 that the function

$$
z \mapsto \operatorname{Det}_{\mathrm{gr}, \theta}^{\prime}\left(\mathcal{B}_{\text {even }}^{(\lambda, \infty)}\left(\nabla_{\gamma(z)}, g\right)\right)
$$

is complex differentiable at 0 .

Let $a \in \mathcal{O}$ be such that $\gamma(a) \in U_{\lambda, \theta}$. By making a change of variables $\zeta=z-0$ we conclude that this function is also complex differentiable at $a$. Hence, this function is holomorphic in $\gamma^{-1}\left(U_{\lambda, \theta}\right)$.

\section{ReFined ANALYTIC TORSION AS A HOLOMORPHIC SECTION}

In this section we show that the refined analytic torsion $\rho_{\text {an }}$ is a nonvanishing holomorphic section of $\mathcal{D} e t$. More precisely, our main result is the following

5.1. Weakly holomorphic section. Recall from [24, p. 148] that a continuous function on a singular space $X$ is called weakly holomorphic if its restriction to the set of regular points of $X$ is holomorphic. Such functions have many properties of analytic functions on $X$. In particular, they are all meromorphic . We refer to [24], [25, §1e] for the properties of the weakly holomorphic functions.

We say that a section $s(\alpha)$ of Det is weakly holomorphic if there exists a weakly holomorphic function $f(\alpha)$ on $\operatorname{Rep}\left(\pi_{1}(M), \mathbb{C}^{n}\right)$, such that $s(\alpha)=f(\alpha) \cdot \tau(\alpha)$.

Theorem 5.2. The refined analytic torsion $\rho_{\text {an }}$ is a weakly holomorphic section of the determinant bundle Det. In particular, the restriction of $\rho_{\text {an }}$ to the set $\operatorname{Rep}_{0}\left(\pi_{1}(M), \mathbb{C}^{n}\right)$ of acyclic representations, viewed as a complex-valued function via the canonical isomorphism

$$
\left.\mathcal{D e t}\right|_{\operatorname{Rep}_{0}\left(\pi_{1}(M), \mathbb{C}^{n}\right)} \simeq \operatorname{Rep}_{0}\left(\pi_{1}(M), \mathbb{C}^{n}\right) \times \mathbb{C},
$$


is a weakly holomorphic function on $\operatorname{Rep}_{0}\left(\pi_{1}(M), \mathbb{C}^{n}\right)$.

5.3. Reduction to a finite dimensional complex. Let $\alpha_{0} \in$ $\operatorname{Rep}\left(\pi_{1}(M), \mathbb{C}^{n}\right)$. Fix a Riemannian metric $g$ on $M$ and a number $\lambda \geq 0$ such that there are no eigenvalues of $\mathcal{B}\left(\nabla_{\alpha_{0}}, g\right)^{2}$ with absolute value equal to $\lambda$. Let $\theta$ be an Agmon angle for $\mathcal{B}\left(\nabla_{\alpha_{0}}, g\right)^{2}$ and let $U_{\lambda, \alpha} \subset \operatorname{Rep}\left(\pi_{1}(M), \mathbb{C}^{n}\right)$ be as in Section 4. By Corollary 4.2 the function $\alpha \mapsto \operatorname{Det}_{\mathrm{gr}, \theta}\left(\mathcal{B}_{\text {even }}^{(\lambda, \infty)}\left(\nabla_{\alpha}, g\right)\right)$ is weakly holomorphic on $U_{\lambda, \theta}$. It follows now from the definition of the refined analytic torsion that to prove Theorem 5.2 it is enough to show that

$$
\alpha \mapsto \rho_{[0, \lambda]} \equiv \rho_{\mathbb{G}_{[0, \lambda]}}\left(\nabla_{\alpha}, g\right)
$$

is a weakly holomorphic section of $\operatorname{Rep}\left(\pi_{1}(M), \mathbb{C}^{n}\right)$. By the definition of the holomorphic structure on the bundle Det, cf. Definition 3.6, this means that the function

$$
\alpha \mapsto \frac{\rho_{\mathbb{G}_{[0, \lambda]}}\left(\nabla_{\alpha}, g\right)}{\tau(\alpha)},
$$

is continuous at $\alpha_{0}$ and is holomorphic at $\alpha_{0}$ if $\alpha_{0}$ is a regular point of $\operatorname{Rep}\left(\pi_{1}(M), \mathbb{C}^{n}\right)$. Here, $\tau(\alpha)$ is defined in (3.10).

If $\alpha_{0}$ is a regular point of $\operatorname{Rep}\left(\pi_{1}(M), \mathbb{C}^{n}\right)$, then by Hartog's theorem, [27, Th. 2.2.8], it is enough to show that for every holomorphic curve $\gamma: \mathcal{O} \rightarrow U_{\lambda, \theta}$, where $\mathcal{O}$ is a connected open neighborhood of 0 in $\mathbb{C}$, the function

$$
f(z):=\frac{\rho_{\mathbb{G}_{[0, \lambda]}}\left(\nabla_{\gamma(z)}, g\right)}{\tau(\gamma(z))}
$$

is complex differentiable at 0 , i.e., there exists $a \in \mathbb{C}$, such that as $z \rightarrow 0$

$$
f(z)=f(0)+z \cdot a+o(z) .
$$

5.4. Choice of a basis. We use the notation introduced in Subsection 4.3. In particular we have a vector bundle $E$, a holomorphic curve $\gamma: \mathcal{O} \rightarrow \operatorname{Rep}\left(\pi_{1}(M), \mathbb{C}^{n}\right)$, and a continuous family of flat connection $\nabla_{\gamma(z)}(z \in \mathcal{O})$ on $E$ such that for each $z \in \mathcal{O}$ the monodromy of $\nabla_{\gamma(z)}$ is equal to $\gamma(z)$. If $\alpha_{0}$ is a regular point of $\operatorname{Rep}\left(\pi_{1}(M), \mathbb{C}^{n}\right)$ then we also assume that

$$
\nabla_{\gamma(z)}=\nabla_{\gamma(0)}+z \cdot \omega+o(z) .
$$

where $o(z)$ is understood in the sense of the Fréchet topology.

Let $\Pi_{[0, \lambda]}(z)(z \in \mathcal{O})$ denote the spectral projection of the operator $\mathcal{B}\left(\nabla_{\gamma(z)}, g\right)^{2}$, corresponding to the set of eigenvalues of $\mathcal{B}\left(\nabla_{\gamma(z)}, g\right)^{2}$, whose absolute value is $\leq \lambda$. Then it follows from the definition of $U_{\lambda}$ that $\Pi_{[0, \lambda]}(z)$ depends continuously on $z$. Moreover, in case when $\alpha_{0}$ is a 
regular point of $\operatorname{Rep}\left(\pi_{1}(M), \mathbb{C}^{n}\right), \Pi_{[0, \lambda]}(z)$ is complex differentiable in $z$. Hence, in this case there exists a bounded operator $R$ on $L_{*}^{2}(M, E \oplus E)$ such that

$$
\Pi_{[0, \lambda]}(z)=\Pi_{[0, \lambda]}(0)+z R+o(z) .
$$

We denote by $\Omega^{\bullet}(z)$ the image of $\Pi_{[0, \lambda]}(z)$. Recall that we denote the dimension of $M$ by $m=2 r-1$. For each $j=0, \ldots, r-1$, fix a basis

$$
\mathbf{w}_{j}=\left\{w_{j}^{1}, \ldots, w_{j}^{l_{j}}\right\}
$$

of $\Omega^{j}(0)$ and set $\mathbf{w}_{m-j}:=\left\{\mathbb{G} w_{j}^{1}, \ldots, \mathbb{G} w_{j}^{l_{j}}\right\}$. To simplify the notation we will write $\mathbf{w}_{m-j}=\mathbb{G} \mathbf{w}_{j}$. Then $\mathbf{w}_{j}$ is a basis for $\Omega^{j}(0)$ for all $j=$ $0, \ldots, m$.

For each $z \in \mathcal{O}, j=0, \ldots, m$, set

$$
\mathbf{w}_{j}(z)=\left\{w_{j}^{1}(z), \ldots, w_{j}^{l_{j}}(z)\right\}:=\left\{\Pi_{[0, \lambda]}(z) w_{j}^{1}, \ldots, \Pi_{[0, \lambda]}(z) w_{j}^{l_{j}}\right\} .
$$

Since $\Pi_{[0, \lambda]}(z)$ depends continuously on $z$, there exists a neighborhood $\mathcal{O}^{\prime} \subset \mathcal{O}$ of 0 , such that $\mathbf{w}_{j}(z)$ is a basis of $\Omega^{j}(z)$ for all $z \in \mathcal{O}^{\prime}$, $j=0, \ldots, m$. Further, since $\Pi_{[0, \lambda]}(z)$ commutes with $\mathbb{G}$, we obtain

$$
\mathbf{w}_{m-j}(z)=\mathbb{G} \mathbf{w}_{j}(z) .
$$

Clearly, $\mathbf{w}_{j}(0)=\mathbf{w}_{j}$ for all $j=0, \ldots, m$.

For each $z \in \mathcal{O}^{\prime}$, the space $\Omega^{\bullet}(z)$ is a subcomplex of $\left(L_{*}^{2}(M, E \oplus\right.$ $\left.E), \mathbb{D}_{\gamma(z)}\right)$. Moreover, the embedding $\Omega^{\bullet}(z) \hookrightarrow L_{*}^{2}(M, E \oplus E)$ is a quasi-isomorphism. It follows from Theorem 2.3 that the cohomology of this compels is canonically isomorphic to

$$
H^{\bullet}\left(M, E_{\gamma(z)}\right) \oplus H^{\bullet}\left(M, \partial M, E_{\gamma(z)}\right) .
$$

Let

$$
\phi_{\Omega^{\bullet}(z)}: \operatorname{Det}\left(\Omega^{\bullet}(z)\right) \longrightarrow \operatorname{Det}\left(H^{\bullet}\left(M, E_{\gamma(z)}\right) \oplus H^{\bullet}\left(M, \partial M, E_{\gamma(z)}\right)\right)
$$

denote the canonical isomorphism, cf. Section 2.4 of [5] . For $z \in$ $\mathcal{O}^{\prime}$, let $w(z) \in \operatorname{Det}\left(\Omega^{\bullet}(z)\right)$ be the element determined by the basis $\mathbf{w}_{1}(z), \ldots, \mathbf{w}_{m}(z)$ of $\Omega^{\bullet}(z)$. More precisely, we introduce

$$
w_{j}(z)=w_{j}^{1}(z) \wedge \cdots \wedge w_{j}^{l_{j}}(z) \in \operatorname{Det}\left(\Omega^{j}(z)\right),
$$

and set

$$
w(z):=w_{0}(z) \otimes w_{1}(z)^{-1} \otimes \cdots \otimes w_{m}(z)^{-1} .
$$

Then, according to Definition 4.3 of [5], it follows from (5.4) that, for all $z \in \mathcal{O}^{\prime}$, the refined torsion of the complex $\Omega^{\bullet}(z)$ is equal to $\phi_{\Omega \bullet}(z)(w(z))$, i.e.,

$$
\rho_{\mathbb{G}_{[0, \lambda]}}\left(\nabla_{\gamma(z)}\right)=\phi_{\Omega \bullet(z)}(w(z))
$$


5.5. Reduction to a family of differentials. Using the basis $\mathbf{w}_{j}(z)$ we define the isomorphism

$$
\psi_{j}(z): \mathbb{C}^{l_{j}} \longrightarrow \Omega_{[0, \lambda]}^{j}(z)
$$

by the formula

$$
\psi_{j}(z)\left(x_{1}, \ldots, x_{l_{j}}\right):=\sum_{k=1}^{l_{j}} x_{k} w_{j}^{k}(z)=\sum_{k=1}^{l_{j}} x_{k} \Pi_{[0, \lambda]}(z) w_{j}^{k} .
$$

We conclude that for each $z \in \mathcal{O}^{\prime}$, the complex $\left(\Omega^{\bullet}(z), \mathbb{D}_{\gamma(z)}\right)$ is isomorphic to the complex

$$
\left(W^{\bullet}, d(z)\right): \quad 0 \rightarrow \mathbb{C}^{l_{0}} \stackrel{d_{0}(z)}{\longrightarrow} \mathbb{C}^{l_{1}} \stackrel{d_{1}(z)}{\longrightarrow} \cdots \stackrel{d_{l-1}(z)}{\longrightarrow} \mathbb{C}^{l_{m}} \rightarrow 0
$$

where

$$
d_{j}(z):=\psi_{j+1}(z)^{-1} \circ \mathbb{D}_{\gamma(z)} \circ \psi_{j}(z), \quad j=0, \ldots, m .
$$

It follows from (5.3) and (5.6) that $d_{j}(z)$ is continuous family of differentials. Moreover, when $\alpha_{0}$ is a regular point of $\operatorname{Rep}\left(\pi_{1}(M), \mathbb{C}^{n}\right)$ it is complex differentiable at 0 , i.e., there exists a $\left(l_{j+1} \times l_{j}\right)$-matrix $A$ such that

$$
d_{j}(z)=d_{j}(0)+z A+o(z) .
$$

Let $\psi(z):=\bigoplus_{j=0}^{d} \psi_{j}(z)$. Since $\mathbb{G}\left(\Omega^{j}(z)\right)=\Omega^{m-j}(z)(j=0, \ldots, m)$, we conclude that $l_{j}=l_{m-j}$. From (5.4) we obtain that

$$
\widehat{\Gamma}:=\psi^{-1}(z) \circ \mathbb{G} \circ \psi(z)
$$

is independent of $z \in \mathcal{O}^{\prime}$ and

$$
\widehat{\Gamma}:\left(x_{1}, \ldots, x_{l_{j}}\right) \mapsto\left(x_{1}, \ldots, x_{l_{j}}\right), \quad j=0, \ldots, m .
$$

It follows from (5.8) and (5.9) that

$$
\rho_{\widehat{\Gamma}}(z)=\rho_{\mathbb{G}_{[0, \lambda]}}\left(\nabla_{\gamma(z)}\right),
$$

where $\rho_{\widehat{\Gamma}}(z)$ denotes the refined torsion of the finite dimensional complex $\left(W^{\bullet}, d(z)\right)$ corresponding to the chirality operator $\widehat{\Gamma}$.

Let $\phi_{W} \bullet(z): \operatorname{Det}\left(W^{\bullet}\right) \rightarrow \operatorname{Det}\left(H^{\bullet}(d(z))\right)$ denote the denote the canonical isomorphism of Section 2.4 of [5] . The standard bases of $\mathbb{C}^{l_{j}}$ $(j=0, \ldots, m)$ define an element $\tilde{w} \in \operatorname{Det}\left(W^{\bullet}\right)$. From (5.10) and the definition of $\rho_{\mathbb{G}}(z)$ we conclude that

$$
\rho_{\mathbb{G}}(z)=\phi_{W} \cdot(z)(\tilde{w}) .
$$


5.6. The acyclic case. To illustrate the main idea of the proof let us first consider the case, when both $H^{\bullet}\left(M, E_{\alpha_{0}}\right)$ and $H^{\bullet}\left(M, \partial M, E_{\alpha_{0}}\right)$ are trivial. Then there exists a neighborhood $\mathcal{O}^{\prime \prime} \subset \mathcal{O}^{\prime}$ of 0 such that $H^{\bullet}\left(M, E_{\gamma(z)}\right)=H^{\bullet}\left(M, \partial M, E_{\gamma(z)}\right)=0$ for all $z \in \mathcal{O}^{\prime \prime}$. Thus the torsion (5.12) is a complex valued function on $\mathcal{O}^{\prime \prime}$. To finish the proof of Theorem 5.2 in this case it remains to show that this function is continuous and, in case when $\alpha_{0}$ is a regular point of $\operatorname{Rep}\left(\pi_{1}(M), \mathbb{C}^{n}\right)$, is complex differentiable at 0 . In view of (5.8), this follows from the following

Lemma 5.7. Let

$$
\begin{aligned}
& \left(C^{\bullet}, \partial(z)\right): \quad 0 \rightarrow \mathbb{C}^{n \cdot k_{0}} \stackrel{\partial_{0}(z)}{\longrightarrow} \quad \mathbb{C}^{n \cdot k_{1}} \stackrel{\partial_{1}(z)}{\longrightarrow} \cdots \\
& \cdots \stackrel{\partial_{m-1}(z)}{\longrightarrow} \mathbb{C}^{n \cdot k_{m}} \rightarrow 0,
\end{aligned}
$$

be a family of acyclic complexes defined for all $z$ in an open set $\mathcal{O} \subset \mathbb{C}$. For any $c \in \operatorname{Det}\left(C^{\bullet}\right)$ the function $z \mapsto \phi_{\left(C^{\bullet}, \partial(z)\right)}(c)$ is continuous if the differentials $\partial_{j}(z)$ are continuous, and is complex differentiable at 0 if $\partial_{j}(z)$ are complex differentiable at 0.

Proof. It is enough to prove the lemma for one particular choice of $c$. To make such a choice let us fix for each $j=0, \ldots, m$ a complement of $\operatorname{Im}\left(\partial_{j-1}(0)\right)$ in $C^{j}$ and a basis $v_{j}^{1}, \ldots, v_{j}^{l_{j}}$ of this complement. Since the complex $C^{\bullet}$ is acyclic, for all $j=0, \ldots, m$, the vectors

$$
\partial_{j-1}(0) v_{j-1}^{1}, \ldots, \partial_{j-1}(0) v_{j-1}^{l_{j-1}}, v_{j}^{1}, \ldots, v_{j}^{l_{j}}
$$

form a basis of $C^{j}$. Let $c \in \operatorname{Det}\left(C^{\bullet}\right)$ be the element defined by these bases. Then, for all $z$ close enough to 0 and for all $j=0, \ldots, m$,

$$
\partial_{j-1}(z) v_{j-1}^{1}, \ldots, \partial_{j-1}(z) v_{j-1}^{l_{j-1}}, v_{j}^{1}, \ldots, v_{j}^{l_{j}}
$$

is also a basis of $C^{j}$. Let $A_{j}(z)(j=0, \ldots, m)$ denote the nondegenerate matrix transforming the basis (5.14) to the basis (5.13). Then, by the definition of the isomorphism $\phi_{\left(C^{\bullet}, \partial(z)\right)}$, cf. $\S 2.4$ of [5],

$$
\phi_{(C \bullet, \partial(z))}(c)=(-1)^{\mathcal{N}\left(C^{\bullet}\right)} \prod_{j=0}^{m} \operatorname{Det}(A(z))^{(-1)^{j}},
$$

where $\mathcal{N}\left(C^{\bullet}\right)$ is the integer defined in formula (2.15) of [5] which is independent of $z$. Clearly, the matrix valued functions $A_{j}(z)$ and, hence, their determinants are continuous if the differentials $\partial_{j}(z)$ are continuous, and are complex differentiable at 0 if $\partial_{j}(z)$ are complex differentiable at 0 . Thus, so is the function $z \mapsto \phi_{(C \bullet, \partial(z))}(c)$. 
5.8. Sketch of the proof of Theorem 5.2 in the non-acyclic case. We now turn to the proof of Theorem 5.2 in the general case. In this subsection we sketch the main ideas of the proof. It is enough to show that the function

$$
f(z):=\frac{\rho_{\mathbb{G}_{[0, \lambda]}}\left(\nabla_{\gamma(z)}, g\right)}{\tau(\gamma(z))}
$$

continuous and, if $\alpha_{0}$ is a regular point of $\operatorname{Rep}\left(\pi_{1}(M), \mathbb{C}^{n}\right)$, is complex differentiable at 0 . Here $\tau$ is the map (3.10). To see this we consider the de Rham integration maps

$$
\begin{aligned}
& J_{z}^{\max }: \Omega_{\max }^{\bullet}\left(M, E_{\gamma(z)}\right) \longrightarrow C^{\bullet}(K, \gamma(z)), \\
& J_{z}^{\min }: \Omega_{\min }^{\bullet}\left(M, E_{\gamma(z)}\right) \longrightarrow C^{\bullet}\left(K, K^{\prime}, \gamma(z)\right) .
\end{aligned}
$$

where the cochain complexes $C^{\bullet}(K, \gamma(z))$ and $C^{\bullet}\left(K, K^{\prime}, \gamma(z)\right)$ are defined in $\S 3$.

The de Rham integration map of $E$-valued differential forms is defined using a trivialization of $E$ over each cell $e_{j}$, and, hence, it depends on the flat connection $\nabla_{\gamma(z)}$, cf. below. More precisely, in the neighborhood of any cell $e$ of $K$, a differential form $f \in \Omega^{\bullet}\left(M, E_{\gamma(z)}\right)$ can be written in the form $f=\sum_{j=1}^{n} f_{j} \otimes v_{j}$, where $f_{j} \in \Omega^{\bullet}(M)$ is a complex-valued differential form and $v_{j}$ is a $\nabla_{\gamma(z)}$-flat section of $E_{\gamma(z)}$ for $j=1, . ., n$. The de Rham integration map is then defined by

$$
J_{z}^{\max } f(e):=\sum_{j=1}^{n}\left(\int_{e} f_{j}\right) v_{j} .
$$

If $f \in \Omega_{\min }^{\bullet}\left(M, E_{\gamma(z)}\right)$ then $J_{z}^{\max } f$ is a well-defined element of the relative cochain complex $C^{\bullet}\left(K, K^{\prime}, \gamma(z)\right)$ and we denote the corresponding map by $J_{z}^{\min }$. Both maps descend to isomorphisms on cohomology [38, $\S 4]$. We write

$$
\begin{aligned}
& \Omega_{d}^{\bullet}\left(M, E_{\alpha}\right):=\Omega_{\min }^{\bullet}\left(M, E_{\alpha}\right) \oplus \Omega_{\max }^{\bullet}\left(M, E_{\alpha}\right), \\
& C_{d}^{\bullet}(K, \alpha):=C^{\bullet}\left(K, K^{\prime}, \alpha\right) \oplus C^{\bullet}(K, \alpha) .
\end{aligned}
$$

Hence we obtain a quasi-isomorphism

$$
J_{z}:=J_{z}^{\max } \oplus J_{z}^{\min }: \Omega_{d}^{\bullet}\left(M, E_{\gamma(z)}\right) \rightarrow C_{d}^{\bullet}(K, \gamma(z)) .
$$

The trivialization $T_{e}(z):\left.E\right|_{e} \rightarrow \mathbb{C}^{n} \times e$ induced by the connection $\nabla_{\gamma(z)}$ is continuous. Moreover, if $\alpha_{0}$ is a regular point of $\operatorname{Rep}\left(\pi_{1}(M), \mathbb{C}^{n}\right)$, it is complex differentiable at $z=0$ by (4.3). Hence so is the de Rham integration map $J_{z}$.

We then consider the restriction $\left.J_{z}\right|_{\Omega} \bullet(z)$ of $J_{z}$ to the finite dimensional complex $\Omega^{\bullet}(z)$ and study the cone complex Cone $\bullet\left(\left.J_{z}\right|_{\Omega \bullet(z)}\right)$ of the map $J_{z}$. This is a finite dimensional acyclic complex with a fixed basis, obtained from the bases of $\Omega^{\bullet}(z)$, defined in $\S 5.4$, and $C_{d}^{\bullet}(K, \gamma(z))$. The 
torsion of this complex is equal to $f(z)$. An application of Lemma 5.7 to this complex proves Theorem 5.2.

In the definition of the integration map $J_{z}$ we have to take into account the fact that the vector bundles $E_{\gamma(z)}$ and $E=E_{\gamma(0)}$ are isomorphic but not equal. The integration map $J_{z}$, cf. Subsection 5.9, is a map from $\Omega_{d}^{\bullet}\left(M, E_{\gamma(z)}\right)$ to the cochain complex $C_{d}^{\bullet}(K, \gamma(z))$, which is not equal to the complex $C_{d}^{\bullet}(K, \gamma(0))$. Fix an Euler structure on $M$. It defines an isomorphism between the complexes $C_{d}^{\bullet}(K, \gamma(0))$ and $C_{d}^{\bullet}(K, \gamma(z))$ which depends on $z$. The study of this isomorphism, which is conducted in Subsection 5.10, is important for the understanding of the properties of $J_{z}$. In particular, it is used to show that in a certain sense $J_{z}$ is complex differentiable at 0 , which implies that the cone complex Cone ${ }^{\bullet}\left(J_{z}\right)$ satisfies the conditions of Lemma 5.7.

5.9. The cochain complex of the bundle E. Fix a CW-decomposition $K=\left\{e_{1}, \ldots, e_{N}\right\}$ of $M$. For each $j=1, \ldots, N$ choose a point $x_{j} \in e_{j}$ and let $E_{x_{j}}$ denote the fiber of $E$ over $x_{j}$. Then the cochain complex $\left(C_{d}^{\bullet}(K, \gamma(z)), \partial_{\bullet}\right)$ may be naturally identified with the complex $\left(C_{\dot{d}}^{\bullet}(K, E), \partial_{\bullet}^{\prime}(z)\right)$ where the $z$-dependence is now fully encoded in the differentials. We use the prime in the notation of the differentials $\partial_{j}^{\prime}$ in order to distinguish them from the differentials of the cochain complex $C_{d}^{\bullet}(K, \gamma(z))$. The differentials $\partial_{j}^{\prime}(z)$ are continuous. Moreover, if $\alpha_{0}$ is a regular point if $\operatorname{Rep}\left(\pi_{1}(M), \mathbb{C}^{n}\right)$ then it follows from (5.2) that $\partial_{j}^{\prime}(z)$ are complex differentiable at 0 , i.e., there exist linear maps $a_{j}$ : $C_{d}^{j}(K, E) \longrightarrow C_{d}^{j+1}(K, E)$, s.t.

$$
\partial_{j}^{\prime}(z)=\partial_{j}^{\prime}(0)+z \cdot a_{j}+o(z), \quad j=1, \ldots, m-1 .
$$

5.10. Relationship with the complex $C_{d}^{\bullet}(K, \gamma(z))$. Recall that for each $z \in \mathcal{O}^{\prime}$ the monodromy representation of $\nabla_{\gamma(z)}$ is equal to $\gamma(z)$. Let $\pi: \widetilde{M} \rightarrow M$ denote the universal cover of $M$ and let $\widetilde{E}=\pi^{*} E$ denote the pull-back of the bundle $E$ to $\widetilde{M}$. Recall that in Subsection 4.3 we fixed a point $x_{*} \in M$. Let $\tilde{x}_{*} \in \widetilde{M}$ be a lift of $x_{*}$ to $\widetilde{M}$ and fix a basis of the fiber $\widetilde{E}_{\tilde{x}_{*}}$ of $\widetilde{E}$ over $x_{*}$. Then, for each $z \in \mathcal{O}^{\prime}$, the flat connection $\nabla_{\gamma(z)}$ identifies $\widetilde{E}$ with the product $\widetilde{M} \times \mathbb{C}^{n}$.

Recall from Subsection 3.7 that the choice of the Euler structure $\varepsilon$ also fixes the lifts $\widetilde{e}_{j}(j=1, \ldots, N)$ of the cell $e_{j}$ fixed in Subsection 3.7. Let $\tilde{x}_{j} \in \widetilde{e}_{j}$ be the lift of $x_{j} \in e_{j}$. Then the trivialization of $\widetilde{E}$ defines a continuous in $z$ family of isomorphisms

$$
S_{z, j}: E_{x_{j}} \simeq \widetilde{E}_{\tilde{x}_{j}} \rightarrow \mathbb{C}^{n}, \quad j=1, \ldots, N, z \in \mathcal{O}^{\prime}
$$


The isomorphisms $S_{z, j}$ depend on the trivialization of $\widetilde{E}$, i.e., on the connection $\nabla_{\gamma(z)}$. The direct sum $S_{z}=\bigoplus_{j} S_{z, j}$ defines an isomorphism $S_{z}: C_{d}^{\bullet}(K, E) \rightarrow C_{d}^{\bullet}(K, \gamma(z))$. If $\alpha_{0}$ is a regular point of $\operatorname{Rep}\left(\pi_{1}(M), \mathbb{C}^{n}\right)$, it follows from (4.3) that $S_{z}$ is complex differentiable at 0 , i.e. for some linear map $s$

$$
S_{z}=S_{0}+z \cdot s+o(z) .
$$

Finally, we consider the morphism of complexes

$$
I_{z}:=S_{z}^{-1} \circ J_{z} \circ \psi(z): W^{\bullet} \rightarrow C^{\bullet}:=C_{d}^{\bullet}(K, E), z \in \mathcal{O}^{\prime} .
$$

This map is complex differentiable at $z=0$ and induces an isomorphism of cohomology.

5.11. The cone complex. The cone complex Cone ${ }^{\bullet}\left(I_{z}\right)$ of the map $I_{z}$ is given by the sequence of vector spaces

$$
\text { Cone }^{j}\left(I_{z}\right):=W^{j} \oplus C_{d}^{j-1}(K, E) \simeq \mathbb{C}^{l_{j}} \oplus \mathbb{C}^{n \cdot k_{j-1}},
$$

$j=0, \ldots, m$, with differentials

$$
\hat{\partial}_{j}(z)=\left(\begin{array}{cc}
d_{j}(z) & 0 \\
I_{z, j} & \partial^{\prime}(\gamma(z))
\end{array}\right)
$$

where $I_{z, j}$ denotes the restriction of $I_{z}$ to $W^{j}$. This is a family of acyclic complexes with differentials $\hat{\partial}_{j}(z)$, which are continuous. If $\alpha_{0}$ is a regular point of $\operatorname{Rep}\left(\pi_{1}(M), \mathbb{C}^{n}\right)$ then $\hat{\partial}_{j}(z)$ are also complex differentiable at 0 . The standard bases of $\mathbb{C}^{l_{j}} \oplus \mathbb{C}^{n \cdot k_{j-1}}$ define an element $c \in \operatorname{Det}\left(\operatorname{Cone}^{\bullet}\left(I_{z}\right)\right)$ which is independent of $z \in \mathcal{O}^{\prime}$. Using the canonical isomorphism of Section 2.4 of [5], we hence obtain for each $z \in \mathcal{O}^{\prime}$ the number $\phi_{\text {Cone }^{\bullet}\left(I_{z}\right)}(c) \in \mathbb{C} \backslash\{0\}$. From the discussion in Subsection 5.8 it follows that this number is equal to the ratio (5.1). Hence, to finish the proof of the Theorem 5.2 it remains to show that the function $z \mapsto \phi_{\text {Cone }{ }^{\bullet}\left(I_{z}\right)}(c)$ is continuous and is complex differentiable at 0 if $\alpha_{0}$ is a regular point of $\operatorname{Rep}\left(\pi_{1}(M), \mathbb{C}^{n}\right)$. This follows immediately from Lemma 5.7.

\section{GLUing FORMUla FOR REFINED ANALYTIC TORSION}

Let $(M, g)$ be a closed oriented Riemannian manifold and $\left(N, g^{N}\right)$ a separating hypersurface, such that $M=M_{1} \cup_{N} M_{2}$. The metric $g$ restricts to Riemannian metrics on the two compact components $M_{1}$ and $M_{2}$. Assume that $g$ is product in an open tubular neighborhood of $N$. 
6.1. The transmission complex $\Omega^{\bullet}\left(M_{1} \# M_{2}, E\right)$. A given representation $\alpha \in \operatorname{Rep}\left(\pi_{1}(M), \mathbb{C}^{n}\right)$ induces a connection $\nabla_{\alpha}$ on a vector bundle $E \equiv E_{\alpha}$, which restricts to well-defined connections on $M_{1,2}$. We denote by $\rho_{i}:=\rho\left(\nabla_{\alpha}, M_{i}\right)$ the refined analytic torsions on $M_{i}, i=1,2$ and by $\rho=\rho\left(\nabla_{\alpha}, M\right)$ for the refined analytic torsion on $M$, cf. (2.13). Let $\iota_{j}: N \hookrightarrow M_{j}$ denote the obvious inclusions, $j=1,2$.

We define the transmission subcomplex of $\Omega^{\bullet}\left(M_{1}, E_{\alpha}\right) \oplus \Omega^{\bullet}\left(M_{2}, E_{\alpha}\right)$ by specifying transmission boundary conditions

$$
\begin{aligned}
\Omega^{\bullet}\left(M_{1} \# M_{2}, E\right) & :=\left\{\left(\omega_{1}, \omega_{2}\right) \in \Omega^{\bullet}\left(M_{1}, E_{\alpha}\right) \oplus \Omega^{\bullet}\left(M_{2}, E_{\alpha}\right) \mid \iota_{1}^{*} \omega_{1}=\iota_{2}^{*} \omega_{2}\right\}, \\
\nabla_{\alpha}\left(\omega_{1}, \omega_{2}\right) & :=\left(\nabla_{\alpha} \omega_{1}, \nabla_{\alpha} \omega_{2}\right) .
\end{aligned}
$$

This defines a complex with eigenforms of the corresponding Laplacian given by the eigenforms of the Hodge-Laplacian on $\left(\Omega^{\bullet}\left(M, E_{\alpha}\right), \nabla_{\alpha}\right)$, cf. [45, Theorem 5.2]. In particular their de Rham cohomologies coincide.

6.2. The fusion map. The splitting $M=M_{1} \cup_{N} M_{2}$ now gives rise to short exact sequences of the associated complexes

$$
\begin{aligned}
& 0 \rightarrow \Omega_{\min }^{\bullet}\left(M_{1}, E\right) \stackrel{\alpha_{1}}{\rightarrow} \Omega^{\bullet}\left(M_{1} \# M_{2}, E\right) \stackrel{\beta_{2}}{\rightarrow} \Omega_{\max }^{\bullet}\left(M_{2}, E\right) \rightarrow 0, \\
& 0 \rightarrow \Omega_{\min }^{\bullet}\left(M_{2}, E\right) \stackrel{\alpha_{2}}{\rightarrow} \Omega^{\bullet}\left(M_{1} \# M_{2}, E\right) \stackrel{\beta_{1}}{\rightarrow} \Omega_{\max }^{\bullet}\left(M_{1}, E\right) \rightarrow 0,
\end{aligned}
$$

where $\alpha_{1}(\omega)=(\omega, 0), \alpha_{2}(\omega)=(0, \omega)$ and $\beta_{j}\left(\omega_{1}, \omega_{2}\right)=\omega_{j}, j=1,2$. The corresponding long exact sequences in cohomology yield canonical isomorphisms, cf. [45]

$$
\begin{aligned}
& \Phi_{1}(\alpha): \operatorname{Det}\left(H^{\bullet}\left(M_{1}, N, E\right)\right) \otimes \operatorname{Det}\left(H^{\bullet}\left(M_{2}, E\right)\right) \rightarrow \operatorname{Det}\left(H^{\bullet}(M, E)\right), \\
& \Phi_{2}(\alpha): \operatorname{Det}\left(H^{\bullet}\left(M_{2}, N, E\right)\right) \otimes \operatorname{Det}\left(H^{\bullet}\left(M_{1}, E\right)\right) \rightarrow \operatorname{Det}\left(H^{\bullet}(M, E)\right) .
\end{aligned}
$$

The fusion isomorphisms, cf. [5, (2.18)] provide canonical identifications

$$
\begin{aligned}
& \mu_{1}: \operatorname{Det}\left(H^{\bullet}\left(M_{1}, N, E\right)\right) \otimes \operatorname{Det}\left(H^{\bullet}\left(M_{1}, E\right)\right) \rightarrow \operatorname{Det}\left(H^{\bullet}\left(\widetilde{\mathcal{D}}_{1}, \mathbb{D}_{1}\right)\right), \\
& \mu_{2}: \operatorname{Det}\left(H^{\bullet}\left(M_{2}, N, E\right)\right) \otimes \operatorname{Det}\left(H^{\bullet}\left(M_{2}, E\right)\right) \rightarrow \operatorname{Det}\left(H^{\bullet}\left(\widetilde{\mathcal{D}}_{2}, \mathbb{D}_{2}\right)\right), \\
& \mu: \operatorname{Det}\left(H^{\bullet}(M, E)\right) \otimes \operatorname{Det}\left(H^{\bullet}(M, E)\right) \rightarrow \operatorname{Det}\left(H^{\bullet}(\widetilde{\mathcal{D}}, \mathbb{D})\right),
\end{aligned}
$$

where the Hilbert complexes $\left(\widetilde{\mathcal{D}}_{j}, \mathbb{D}_{j}\right)$ are defined in Definition 2.6, with the lower index $j$ referring to the underlying manifold $M_{j}, j=1,2$. The Hilbert complex $(\widetilde{\mathcal{D}}, \mathbb{D})$ is defined over $M$. We put

$$
\begin{aligned}
\Phi \equiv \Phi(\alpha) & =\mu \circ\left(\Phi_{1}(\alpha) \otimes \Phi_{2}(\alpha)\right) \circ\left(\mu_{1}^{-1} \otimes \mu_{2}^{-1}\right): \\
& \operatorname{Det}\left(H^{\bullet}\left(\widetilde{\mathcal{D}}_{1}, \mathbb{D}_{1}\right)\right) \otimes \operatorname{Det}\left(H^{\bullet}\left(\widetilde{\mathcal{D}}_{2}, \mathbb{D}_{2}\right)\right) \rightarrow \operatorname{Det}\left(H^{\bullet}(\widetilde{\mathcal{D}}, \mathbb{D})\right) .
\end{aligned}
$$

If $\alpha \in \operatorname{Rep}\left(\pi_{1}(M), \mathbb{C}^{n}\right)$ is unitary, [45, Theorem 10.6] asserts

$$
\Phi\left(\rho_{1} \otimes \rho_{2}\right)=K \cdot \rho,
$$


where

$$
K \equiv K(\alpha)=\sigma 2^{\chi(N)} e^{\pi i\left(\eta\left(\mathcal{B}_{\alpha}, M\right)-\eta\left(\mathcal{B}_{\alpha}, M_{1}\right)-\eta\left(\mathcal{B}_{\alpha}, M_{2}\right)\right)} .
$$

Here $\chi(N)$ stands for the Euler characteristic of $\left(N, E_{\alpha}\lceil N)\right.$ and the $\eta$-invariants $\eta\left(\mathcal{B}_{\alpha}, M\right), \eta\left(\mathcal{B}_{\alpha}, M_{1}\right)$ and $\eta\left(\mathcal{B}_{\alpha}, M_{2}\right)$ are defined in terms of the even parts of the corresponding odd signature operators. The sign $\sigma$ is determined by formula (8.4) of [45]. Note that the sign depends on the dimensions of various cohomology spaces and is related to the sign convention used in defining the fusion isomorphism of determinant lines, see $[5, \S 2]$ for a detail discussion of the sign conventions. Note also that the Euler characteristic $\chi(N)$ depends only on the rank $n$ of the representation $\alpha$ but not on the particular choice of $\alpha \in \operatorname{Rep}\left(\pi_{1}(M), \mathbb{C}^{n}\right)$.

The $\eta$-invariants $\eta\left(\mathcal{B}_{\alpha}, M\right), \eta\left(\mathcal{B}_{\alpha}, M_{1}\right)$ and $\eta\left(\mathcal{B}_{\alpha}, M_{2}\right)$ are not necessarily continuous functions of $\alpha$ : they have integer jumps when some eigenvalues of the odd signature operator cross zero. Hence, $K^{2}(\alpha)$ is a continuous function of $\alpha$. Moreover, below we show that $K^{2}$ extends to a weakly holomorphic function on the space $\operatorname{Rep}\left(\pi_{1}(M), \mathbb{C}^{n}\right)$ of representations.

\subsection{The gluing formula for some non-unitary representations.}

The main result of this section is the following extension of (6.2) to some class of non-unitary representations.

Theorem 6.4. Let $(M, g)$ be a closed oriented Riemannian manifold of odd dimension, and $N$ a separating hypersurface such that $M=M_{1} \cup_{N}$ $M_{2}$, and $g$ is product in an open tubular neighborhood of $N$. Assume that $\mathcal{C} \subset \operatorname{Rep}\left(\pi_{1}(M), \mathbb{C}^{n}\right)$ is a connected component and $\alpha_{0} \subset \mathcal{C}$ is a unitary representation which is a regular point of the complex analytic set $\mathcal{C}$. For any representation $\alpha \in \operatorname{Rep}\left(\pi_{1}(M), \mathbb{C}^{n}\right)$ denote by $\rho(\alpha)$ and $\rho_{j}(\alpha)$ the refined analytic torsions on $M$ and $M_{j}, j=1,2$. Then for any $\alpha \in \mathcal{C}$ we have

$$
\Phi\left(\rho_{1}(\alpha) \otimes \rho_{2}(\alpha)\right)= \pm K(\alpha) \cdot \rho(\alpha) .
$$

The rest of this section is occupied with the proof of Theorem 6.4, which is based on an analytic continuation technique, cf. [9, §6.4]. First we need the following proposition.

Proposition 6.5. $K(\alpha)^{2}$ is a weakly holomorphic function on the complex analytic space $\operatorname{Rep}\left(\pi_{1}(M), \mathbb{C}^{n}\right)$.

Proof. We need to show that $\exp \left(2 i \pi \eta\left(\mathcal{B}_{\alpha}, M_{i}\right)\right), \quad(i=1,2)$ and $\exp \left(2 i \pi \eta\left(\mathcal{B}_{\alpha}, M\right)\right)$ are weekly holomorphic functions of $\alpha$. 
Denote by $\mathcal{B}_{\alpha, j}(j=1,2)$ the odd signature operator of the complex $\left(\widetilde{\mathcal{D}}_{j}, \mathbb{D}_{j}\right)$ and by $\mathcal{B}_{\alpha}$ the odd signature operator of the comples $(\widetilde{\mathcal{D}}, \mathbb{D})$. With this notation we have

$$
\eta\left(\mathcal{B}_{\alpha}, M_{j}\right)=\eta\left(\mathcal{B}_{\alpha, j}\right), \quad \eta\left(\mathcal{B}_{\alpha}, M\right)=\eta\left(\mathcal{B}_{\alpha}\right) .
$$

Fix $\alpha_{0} \in \operatorname{Rep}\left(\pi_{1}(M), \mathbb{C}^{n}\right)$ and a number $\lambda>0$ such that the spectra of the operators $\mathcal{B}_{\alpha_{0}}$ and $\mathcal{B}_{\alpha_{0}, j}(j=1,2)$ do not intersect the circle $\{z \in$ $\mathbb{C}:|z|=\lambda\}$. There exists a neighborhood $U_{\lambda}$ of $\alpha_{0}$ in $\operatorname{Rep}\left(\pi_{1}(M), \mathbb{C}^{n}\right)$ such that for all $\alpha \in U_{\lambda}$ the spectra of the operators $\mathcal{B}_{\alpha}$ and $\mathcal{B}_{\alpha, j}$ $(j=1,2)$ do not intersect the circle $\{z \in \mathbb{C}:|z|=\lambda\}$. Then (cf. formula (4-1) of [44])

$$
\eta\left(\mathcal{B}_{\alpha, j}\right)-\eta\left(\mathcal{B}_{\alpha, j}^{(\lambda, \infty)}\right) \in \frac{1}{2} \mathbb{Z}, \quad \eta\left(\mathcal{B}_{\alpha}\right)-\eta\left(\mathcal{B}_{\alpha}^{(\lambda, \infty)}\right) \in \frac{1}{2} \mathbb{Z} .
$$

Notice that the functions $\eta\left(\mathcal{B}_{\alpha, j}^{(\lambda, \infty)}\right)$ and $\eta\left(\mathcal{B}_{\alpha}^{(\lambda, \infty)}\right)$ are continuous on $U_{\lambda}$. The functions $\eta\left(\mathcal{B}_{\alpha, j}\right)$ and $\eta\left(\mathcal{B}_{\alpha}\right)$ are not necessarily continuous, but might have integer jumps. Hence, it follows from (6.4) that $\eta\left(\mathcal{B}_{\alpha, j}\right)-$ $\eta\left(\mathcal{B}_{\alpha, j}^{(\lambda, \infty)}\right)$ and $\eta\left(\mathcal{B}_{\alpha}\right)-\eta\left(\mathcal{B}_{\alpha}^{(\lambda, \infty)}\right)$ are constants modulo $\mathbb{Z}$. We conclude that

$$
\exp \left(2 i \pi \eta\left(\mathcal{B}_{\alpha, j}\right)-\exp \left(2 i \pi \eta\left(\mathcal{B}_{\alpha, j}^{(\lambda, \infty)}\right), \quad j=1,2,\right.\right.
$$

and

$$
\exp \left(2 i \pi \eta\left(\mathcal{B}_{\alpha}\right)-\exp \left(2 i \pi \eta\left(\mathcal{B}_{\alpha}^{(\lambda, \infty)}\right)\right.\right.
$$

are constant functions on $U_{\lambda}$. Hence, it suffices to show that the functions $\exp \left(2 i \pi \eta\left(\mathcal{B}_{\alpha, j}^{(\lambda, \infty)}\right),(i=1,2)\right.$ and $\exp \left(2 i \pi \eta\left(\mathcal{B}_{\alpha}^{(\lambda, \infty)}\right)\right)$ are weakly holomorphic in a neighborhood of $\alpha_{0}$.

Let $\theta$ be an Agmon angle for the operators $B_{\alpha_{0}}$ and $B_{\alpha_{0}, j}$. Then there exists a neighborhood $U_{\lambda, \theta} \subset U_{\lambda}$ of $\alpha_{0}$, such that for all $\alpha \in U_{\lambda, \theta}$ $\theta$ is an Agmon angle for $B_{\alpha}$ and $B_{\alpha, j}$. By [44, (4.6)] we obtain

$\operatorname{Det}_{g r, \theta}^{\prime}\left(\mathcal{B}_{\alpha, j, \text { even }}^{(\lambda, \infty)}\right)=\exp \left(\xi_{\lambda}\left(\alpha, M_{j}\right)-i \pi \xi_{\lambda}^{\prime}\left(\alpha, M_{j}\right)-i \pi \eta\left(\mathcal{B}_{\alpha}^{(\lambda, \infty)}, M_{j}\right)\right)$

where we have set

$$
\begin{aligned}
& \xi_{\lambda}\left(\alpha, M_{j}\right):=\left.\frac{1}{2} \sum_{k=0}^{m}(-1)^{k} \cdot k \cdot \frac{d}{d s}\right|_{s=0} \zeta_{2 \theta}\left(s, \mathcal{B}_{\alpha, j}^{2} \uparrow \widetilde{\mathcal{D}}_{j,(\lambda, \infty)}^{k}\right), \\
& \xi_{\lambda}^{\prime}\left(\alpha, M_{j}\right):=\frac{1}{2} \sum_{k=0}^{m}(-1)^{k} \cdot k \cdot \zeta_{2 \theta}\left(s=0, \mathcal{B}_{\alpha, j}^{2}\left\lceil\widetilde{\mathcal{D}}_{j,(\lambda, \infty)}^{k}\right) .\right.
\end{aligned}
$$

The graded determinant $\operatorname{Det}_{g r, \theta}^{\prime}\left(\mathcal{B}_{\alpha, j, \text { even }}\right)$ is weakly holomorphic in $U_{\lambda, \theta}$ by Corollary 4.2. The fact that $\exp \left(2 \xi\left(\alpha, M_{j}\right)\right)$ and 
$\exp \left(2 i \pi \xi^{\prime}\left(\alpha, M_{j}\right)\right)$ are weakly holomorphic follows similarly from the variational formula

$$
\begin{aligned}
&\left.\frac{d}{d t}\right|_{t=0} \zeta_{2 \theta}\left(s, \mathcal{B}_{\kappa(t)}^{2} \uparrow \widetilde{\mathcal{D}}_{j,(\lambda, \infty)}^{k}\right) \\
&=-s \cdot \operatorname{Tr}\left(\frac { d } { d t } | _ { t = 0 } \mathcal { B } _ { \kappa ( t ) } ^ { 2 } \lceil \widetilde { \mathcal { D } } _ { j , ( \lambda , \infty ) } ^ { k } ) \left(\mathcal{B}_{\kappa(0)}^{2}\left\lceil\widetilde{\mathcal{D}}_{j,(\lambda, \infty)}^{k}\right)^{-s-1} .\right.\right.
\end{aligned}
$$

As a consequence, $\exp \left(2 i \pi \eta\left(\mathcal{B}_{\alpha, j}^{(\lambda, \infty)}\right)\right)(j=1,2)$ are weakly holomorphic. Similarly, on proves that $\exp \left(2 i \pi \eta\left(\mathcal{B}_{\alpha}^{(\lambda, \infty)}\right)\right)$ is weakly holomorphic.

6.6. An analytic continuation. The set of unitary representations is the fixed point set of the anti-holomorphic involution

$$
\tau: \operatorname{Rep}\left(\pi_{1}(M), \mathbb{C}^{n}\right) \rightarrow \operatorname{Rep}\left(\pi_{1}(M), \mathbb{C}^{n}\right), \quad \tau: \alpha \mapsto \alpha^{\prime},
$$

where $\alpha^{\prime}$ denotes the representation dual to $\alpha$. Hence, it is a totally real submanifold of $\operatorname{Rep}\left(\pi_{1}(M), \mathbb{C}^{n}\right)$ whose real dimension is equal to $\operatorname{dim}_{\mathbb{C}} \mathcal{C}$, cf. [26, Proposition 3]. In particular there is a holomorphic coordinates system $\left(z_{1}, \ldots, z_{r}\right)$ near $\alpha_{0}$ such that the unitary representations form a real neighborhood of $\alpha_{0}$, i.e. the set $\operatorname{Im} z_{1}=\ldots=$ $\operatorname{Im} z_{r}=0$. Therefore, cf. [39, p. 21], we obtain the following

Proposition 6.7. If two holomorphic functions coincide on the set of unitary representations they also coincide on $\mathcal{C}$.

6.8. The proof of Theorem 6.4. By Theorem 5.2 the refined analytic torsions $\rho_{1}, \rho_{2}$ and $\rho$ define holomorphic sections on the corresponding determinant line bundles. The canonical isomorphism $\Phi$ defines a bilinear map between holomorphic determinant line bundles and hence maps holomorphic sections to holomorphic sections. Let us denote by $f(\alpha)$ the unique complex valued function of $\alpha$ such that

$$
\Phi\left(\rho_{1} \otimes \rho_{2}\right)=f(\alpha) \cdot \rho,
$$

holds for all $\alpha \in \mathcal{C}$. Since both $\Phi\left(\rho_{1} \otimes \rho_{2}\right)$ and $\rho$ are holomorphic sections of the determinant line bundle, $f(\alpha)$ is a holomorphic function.

It follows from (6.2) that

$$
K(\alpha)^{2}=f(\alpha)^{2}
$$

for all unitary representations in $\mathcal{C}$. By Proposition $6.5, K(\alpha)^{2}$ is a holomorphic function. Hence, we obtain from Proposition 6.7 that (6.5) holds for all $\alpha \in \mathcal{C}$. 
Remark 6.9. The gluing formula for refined analytic torsion may be used to prove a gluing result for the Ray-Singer torsion norm on connected components of the representation variety that contain a unitary point.

\section{Gluing FORMula fOR Ray-Singer Analytic torsion}

We continue in the previously outlined setup of a closed oriented Riemannian manifold $(M, g)$ and a separating hypersurface $\left(N, g^{N}\right)$, such that $M=M_{1} \cup_{N} M_{2}$. Consider a representation $\alpha \in \operatorname{Rep}\left(\pi_{1}(M), \mathbb{C}^{n}\right)$ and the corresponding flat vector bundle $(E, \nabla)$. Fix a Hermitian metric $h_{0}$ on $E$, of product type near $N$. Even if the metric structures $\left(g, h_{0}\right)$ are product near $N$, the connection $\nabla$ need not be product near $N$, so that the resulting Laplacian does not have a product structure near the separating hypersurface and hence a gluing theorem for RaySinger analytic torsion cannot be obtained from the results of [34], [46] and [33].

Before we proceed, let us make some chronological remarks on that topic. Vishik [46] was first to prove the gluing formula for analytic torsion given a unitary representation without using the theorem of Cheeger [17] and Müller [35]. Though it was not explicitly stated in [46], the assumption of a unitary representation is obsolete once a connection is in temporal gauge ${ }^{1}$ near $N$. Under the assumption of temporal gauge and product metric structures, the Hodge Laplacian is of product type near $N$ and the Vishik's argument goes through. Recently, Lesch [33] provided an excellent discussion of the gluing formula for possibly non-compact spaces, extending the result of Vishik, and stating clearly that the proof requires only product metric structures and a connection in temporal gauge near the cut, rather than unitariness of the representation.

A quite general proof of the gluing formula for general representations and without assuming product metric structures, was provided by Brüning and Ma [12]. They derive a gluing formula by relating the Ray-Singer and the Milnor torsions, in odd and also in the evendimensional case. In this section we present a different proof of their result in odd dimensions, as a consequence of [33] and the Brüning-Ma anomaly formula in [11]. To make the main idea of our alternative argument clear, we restrict ourselves to the situation, when $\partial M=\varnothing$.

\footnotetext{
${ }^{1}$ The notion and properties of temporal gauge are recalled in the Appendix.
} 
Proposition 7.1. Consider two Hermitian metrics $h_{0}, h_{1}$ on a fixed flat vector bundle $(E, \nabla)$ over a compact oriented odd-dimensional Riemannian manifold $(K, g), j=1,2$. If $\partial K \neq \varnothing$, assume that $h_{0}, h_{1}$ coincide over $Y=\partial K$. Fix either relative or absolute boundary conditions at $Y$ for the Hodge Laplacian and denote by $\|\cdot\|_{\left(g, h_{j}\right)}^{\mathrm{RS}}, j=0,1$, the corresponding Ray-Singer analytic torsion norms. Then ${ }^{2}$

$$
\|\cdot\|_{\left(g, h_{0}\right)}^{\mathrm{RS}}=\|\cdot\|_{\left(g, h_{1}\right)}^{\mathrm{RS}} .
$$

Proof. The metric anomaly, identified in [11] is expressed in terms of the Levi-Civita connections $\nabla^{T K}$ and $\nabla^{T Y}$ on $K$ and its boundary $Y$, the respective representatives $e\left(T K, \nabla^{T K}\right), e(T Y, \nabla T Y)$ of the Euler classes of $T K, T Y$ in Chern-Weil theory, and the quotient $\|\cdot\|_{h_{0}} /\|\cdot\|_{h_{1}}$ between the metrics on $\operatorname{det} E$, induced by $h_{0}$ and $h_{1}$.

Since $\operatorname{dim} K$ is odd, $e\left(T K, \nabla^{T K}\right)=0$. If $h_{0}, h_{1}$ coincide over $Y$, $\log \|\cdot\|_{h_{0}} /\|\cdot\|_{h_{1}}=0$ over $Y$, so that the statement follows from [11, Theorem 0.1, (0.5)].

The main idea now is the reduction to the setup of a connection in temporal gauge near $N$. A connection $\nabla$ is in temporal gauge in an open neighborhood $\mathscr{U}=(-\epsilon, \epsilon) \times N$ of $N$, if $\nabla=\pi^{*} \nabla^{N}$ for some flat connection $\nabla^{N}$ on $E_{N}$, where $\pi: \mathscr{U} \rightarrow N$ is the natural projection onto the second factor. Proposition 8.2 below asserts that in fact every connection is gauge equivalent to a connection in temporal gauge.

We denote the corresponding gauge transformation by $\gamma$. The gauge transformed connection is given by $\nabla_{\gamma}=\gamma \nabla \gamma^{-1}$. We set for any $u, v \in \Gamma(M, E)$

$$
h_{\gamma}(u, v):=h_{0}(\gamma u, \gamma v) .
$$

This defines a new Hermitian metric on $E$ that coincides with $h$ over $N$, since $\gamma$ acts as identity over $N$. By construction, $\gamma$ induces an isometry

$$
\gamma: L_{*}^{2}\left(M, E ; g, h_{\gamma}\right) \rightarrow L_{*}^{2}\left(M, E ; g, h_{0}\right) .
$$

The following theorem is a result by Lesch [33], cf. Vishik [46].

Theorem 7.2. Let $(M, g)$ be a closed oriented Riemannian manifold of odd dimension, and $N$ a separating hypersurface such that $M=$ $M_{1} \cup_{N} M_{2}$, and $g$ is product in an open tubular neighborhood of $N$. Consider flat Hermitian vector bundle $\left(E, \nabla_{\gamma}, h_{0}\right)$. Denote by $\|\cdot\|^{\mathrm{RS}}$ and $\|\cdot\|_{M_{i}}^{\mathrm{RS}}$ the corresponding Ray-Singer norms on $M$ and $M_{i}, i=1,2$, respectively. The Ray-Singer norm on $M_{1}$ is defined with respect to

\footnotetext{
${ }^{2}$ There is no product structure assumption on $g$ and $h_{j}, j=0,1$.
} 
relative boundary conditions, while on $M_{2}$ we pose absolute boundary conditions. Then

$$
\log \frac{\|\cdot\|_{M}^{\mathrm{RS}}}{\Phi_{1}\left(\|\cdot\|_{M_{1}}^{\mathrm{RS}} \otimes\|\cdot\|_{M_{2}}^{\mathrm{RS}}\right)}=\frac{1}{2} \chi(N) \log 2 .
$$

Corollary 7.3. Let the Ray-Singer torsion norms $\|\cdot\|^{\mathrm{RS}}$ and $\|\cdot\|_{M_{i}}^{\mathrm{RS}}$ be defined with respect to the flat connection $\nabla$ and any Hermitian metric $h$ on $E$. Then

$$
\log \frac{\|\cdot\|_{M}^{\mathrm{RS}}}{\Phi_{1}\left(\|\cdot\|_{M_{1}}^{\mathrm{RS}} \otimes\|\cdot\|_{M_{2}}^{\mathrm{RS}}\right)}=\frac{1}{2} \chi(N) \log 2 .
$$

Proof. The Laplacian $\Delta_{\gamma}$ on $E_{\gamma}=\left(E, \nabla_{\gamma}, h_{0}\right)$ is related to the Laplacian $\Delta$ on $E_{\alpha}=\left(E, \nabla, h_{\gamma}\right)$ by the unitary transformation $\gamma$ with $\Delta_{\gamma}=\gamma \circ \Delta \circ \gamma^{-1}$. Hence, $\gamma$ induces a map between the harmonic forms of $\Delta$ and $\Delta_{\gamma}$, and hence also between the corresponding determinant lines, which we also denote by $\gamma$. By construction, we find

$$
\|\gamma(\cdot)\|_{E_{\gamma}}^{\mathrm{RS}}=\|\cdot\|_{E_{\alpha}}^{\mathrm{RS}}
$$

where we indicate the dependence on the vector bundle by the subindex and omit the reference to the underlying manifold, since the relation holds both on $M$ and $M_{i}, i=1,2$. The isometric identification $\gamma$ commutes with the maps in the (6.1). Hence $\Phi_{1} \circ(\gamma \otimes \gamma)=\gamma \circ \Phi_{1}$, and by Theorem 7.2, we find

$$
\log \frac{\|\cdot\|_{\left(M, E_{\alpha}\right)}^{\mathrm{RS}}}{\Phi_{1}\left(\|\cdot\|_{\left(M_{1}, E_{\alpha}\right)}^{\mathrm{RS}} \otimes\|\cdot\|_{\left(M_{2}, E_{\alpha}\right)}^{\mathrm{RS}}\right)}=\frac{1}{2} \chi(N) \log 2 .
$$

This is a gluing theorem for any complex representation $\alpha$, possibly non-unitary. A priori, however, this relation holds for the specific Hermitian metric $h_{\gamma}$ on $\left(E, \nabla_{\alpha}\right)$. Since $\gamma \uparrow N=\mathrm{id}$, the metrics $h_{0}, h_{\gamma}$ coincide over $N$ and hence, by Proposition 7.1 the gluing formula (7.2) holds for any representation $\alpha$ and any choice of a Hermitian metric $h$ on $\left(E, \nabla_{\alpha}\right)$.

The Riemannian metric $g$ is still assumed to be product near $N$, so that variation of $g$ leads to additional anomaly terms, cf. [11, Theorem 0.1 .

\section{Appendix: Temporal Gauge Transformation}

In this section we recall the notion of a connection in temporal gauge and review some properties of these connections, cf. [45]. In particular, we show that any flat connection is gauge equivalent to a connection in temporal gauge, cf. Proposition 8.2. Consider a closed oriented 
Riemannian manifold $\left(M, g^{M}\right)$ of dimension $m$ and a vector bundle $E$ with structure group $G \subset G L(n, \mathbb{C})$. Denote the principal $G$-bundle associated to $E$ by $P$, where $G$ acts on $P$ from the right.

Consider a hypersurface $N \subset M$ and its collar neighborhood $U \cong$ $(-\epsilon, \epsilon) \times N$. We view the restrictions $\left.P\right|_{U},\left.P\right|_{N}$ as $G$-bundles, where the structure group can possibly be reduced to a subgroup of $G$. Let $\pi:(-\epsilon, \epsilon) \times \partial X \rightarrow \partial X$ be the natural projection onto the second component. Then $\left.\left.E\right|_{U} \cong \pi^{*} E\right|_{N}$ and for the associated principal bundles $\left.\left.\left.P\right|_{U} \cong \pi^{*} P\right|_{N} \stackrel{f}{\rightarrow} P\right|_{N}$, where $f$ is the principal bundle homomorphism, covering $\pi$, with the associated homomorphism of the structure groups being the identity automorphism.

Definition 8.1. We call a flat connection $\omega$ on $P$ a connection in temporal gauge over $U$, if there exists a flat connection $\omega_{N}$ on $P_{N}$ such that $\left.\omega\right|_{U}=f^{*} \omega_{N}$ over the collar neighborhood $U$. Similar condition defines a covariant derivative in temporal gauge.

We now explain the notion of temporal gauge in local terms. Let $\omega_{N}$ denote a flat connection one-form on $\left.P\right|_{N}$. Then $\omega_{U}:=f^{*} \omega_{N}$ gives a connection one-form on $\left.P\right|_{U}$ which is flat again. Let $\left\{\widetilde{U}_{\beta}, \widetilde{\Phi}_{\beta}\right\}_{\beta}$ be a set of local trivializations for $\left.P\right|_{N}$. Then $\left.\left.P\right|_{U} \cong \pi^{*} P\right|_{N}$ is trivialized over the local neighborhoods $U_{\beta}:=(-\epsilon, \epsilon) \times \widetilde{U}_{\beta}$ with the induced trivializations $\Phi_{\beta}$. For any $y \in \widetilde{U}_{\beta}$, normal variable $x \in(-\epsilon, \epsilon)$ and for $e \in G$ being the identity matrix we put

$$
\widetilde{s}_{\beta}(y):=\widetilde{\Phi}_{\beta}^{-1}(y, e), \quad s_{\beta}(x, y):=\Phi_{\beta}^{-1}((x, y), e) .
$$

These local sections define local representations for $\omega_{U}$ and $\omega_{N}$

$$
\begin{gathered}
\widetilde{\omega}^{\beta}:=\widetilde{s}_{\beta}^{*} \omega_{N} \in \Omega^{1}\left(\widetilde{U}_{\beta}, \mathcal{G}\right), \\
\omega^{\beta}:=s_{\beta}^{*} \omega_{U} \in \Omega^{1}\left(U_{\beta}, \mathcal{G}\right),
\end{gathered}
$$

where $\mathcal{G}$ denotes the Lie algebra of $G$. Consider local coordinates $y=$ $\left(y_{1}, . ., y_{m-1}\right)$ on $\widetilde{U}_{\beta}$. Then

$$
\begin{aligned}
& \widetilde{\omega}^{\beta}=\sum_{i=1}^{n} \omega_{i}^{\beta}(y) d y_{i}, \quad \omega^{\beta}=\omega_{0}^{\beta}(x, y) d x+\sum_{i=1}^{n} \omega_{i}^{\beta}(x, y) d y_{i}, \\
& \text { with } \quad \omega_{0}^{\beta} \equiv 0, \text { and } \omega_{i}^{\beta}(x, y) \equiv \omega_{i}^{\beta}(y) .
\end{aligned}
$$

Proposition 8.2. Any flat connection on the principal bundle $P$ is gauge equivalent to a flat connection in temporal gauge.

Proof. By a partition of unity argument it suffices to discuss the problem locally over $U_{\beta}$. Let $\omega$ be a flat connection on $\left.P\right|_{U}$. Let $\gamma \in$ $\operatorname{Aut}\left(\left.P\right|_{U}\right)$ be any gauge transformation on $\left.P\right|_{U}$ and $\gamma^{\beta}$ the corresponding local representation. Denote the gauge transform of $\omega$ under $\gamma$ by 
$\omega_{\gamma}$. The local $\mathcal{G}$-valued one-forms $\omega^{\beta}, \omega_{\gamma}^{\beta}$ are related in correspondence to the transformation law of connections by

$$
\omega_{\gamma}^{\beta}=\left(\gamma^{\beta}\right)^{-1} \cdot \omega^{\beta} \cdot \gamma^{\beta}+\left(\gamma^{\beta}\right)^{-1} d \gamma^{\beta},
$$

where the action - is the multiplication of matrices $(G \subset G L(n, \mathbb{C}))$, after evaluation at a local vector field and a base point in $U_{\beta}$.

The local one form $\omega^{\beta}$ can be written as

$$
\omega^{\beta}=\omega_{0}^{\beta}(x, y) d x+\sum_{i=1}^{n} w_{i}^{\beta}(x, y) d y_{i} .
$$

Our task is to identify the correct gauge transformation $\gamma$, so that $\omega$ is temporal gauge, cf. (8.1). For this reason we consider the following initial value problem with parameter $y \in \widetilde{U}_{\beta}$

$$
\begin{aligned}
\partial_{x} \gamma^{\beta}(x, y) & =-\omega_{0}^{\beta}(x, y) \gamma^{\beta}(x, y), \\
\gamma^{\beta}(0, y) & =\mathbf{1} \in G L(n, \mathbb{C}) .
\end{aligned}
$$

The solution to (8.2) is given by an integral curve of the time dependent vector field $V_{x, y}^{\beta}$ on $G$, parametrized by $x \in(-\epsilon, \epsilon)$, such that for any $u \in G$

$$
V_{x, y}^{\beta} u:=-\left(R_{u}\right)_{*} \omega_{0}^{\beta}(x, y)=-\omega_{0}^{\beta}(x, y) \cdot u,
$$

where $R_{u}$ is the right multiplication on $\gamma$ and the second equality follows from the fact that $G \subset G L(n, \mathbb{C})$ is a matrix Lie group. The corresponding integral curve $\gamma^{\beta}(x, y)$ with $\gamma^{\beta}(0, y)=\mathbf{1} \in G$ is $G$-valued and the unique solution to (8.2).

We now compute for the gauge transformed connection $\omega_{\gamma}$

$$
\begin{aligned}
\omega_{\gamma}^{\beta} & =\left(\gamma^{\beta}\right)^{-1} \cdot \omega^{\beta} \cdot \gamma^{\beta}+\left(\gamma^{\beta}\right)^{-1} d \gamma^{\beta} \\
& =\left(\gamma^{\beta}\right)^{-1} \cdot \omega_{0}^{\beta} \cdot \gamma^{\beta} d x+\sum_{i=1}^{n}\left(\gamma^{\beta}\right)^{-1} \cdot \omega_{i}^{\beta} \cdot \gamma^{\beta} d y_{i} \\
& +\left(\gamma^{\beta}\right)^{-1} \partial_{x} \gamma^{\beta} d x+\sum_{i=1}^{n}\left(\gamma^{\beta}\right)^{-1} \partial_{y_{i}} \gamma^{\beta} d y_{i} \\
& =\sum_{i=1}^{n}\left(\gamma^{\beta}\right)^{-1} \cdot \omega_{i}^{\beta} \cdot \gamma^{\beta} d y_{i}+\sum_{i=1}^{n}\left(\gamma^{\beta}\right)^{-1} \partial_{y_{i}} \gamma^{\beta} d y_{i} .
\end{aligned}
$$

where in the last equality we cancelled two summands due to $\gamma^{\beta}$ being the solution to (8.2). We now use the fact that $\omega$ is a flat connection. A gauge transformation preserves flatness, so $\omega_{\gamma}$ is flat again. Put

$$
\omega_{\gamma}^{\beta}=\omega_{\gamma, 0}^{\beta}(x, y) d x+\sum_{i=1}^{n} \omega_{\gamma, i}^{\beta}(x, y) d y_{i},
$$


where by the previous calculation

$$
\omega_{\gamma, 0}^{\beta} \equiv 0, \quad \omega_{\gamma, i}^{\beta} \equiv\left(\gamma^{\beta}\right)^{-1} \cdot \omega_{i}^{\beta} \cdot \gamma^{\beta}+\left(\gamma^{\beta}\right)^{-1} \partial_{y_{i}} \gamma^{\beta} .
$$

Flatness of $\omega_{\gamma}$ implies

$$
\partial_{x} \omega_{\gamma, i}^{\beta}(x, y)=\partial_{y_{i}} \omega_{\gamma, 0}^{\beta}(x, y)=0 .
$$

Hence the gauge transformed connection is indeed in temporal gauge. This completes the proof.

\section{REFERENCES}

[1] Baird, T. and Ramras, D., Smoothing maps into algebraic sets and spaces of flat connections, Geom. Dedicata 174 (2015), 359-374. MR 3303057

[2] Bismut, J.-M. and Zhang, W. An extension of a theorem by Cheeger and Müller. With an appendix by Francois Laudenbach. Asterisque No. 205, (1992), 235 pp.

[3] Bismut, J.-M. and Goette, S. Families torsion and Morse functions. Asterisque No. 275, (2001), 293 pp.

[4] Braverman, M. and Kappeler, T. Ray-Singer type theorem for the refined analytic torsion. J. Funct. Anal. 243 (2007), no. 1, 232-256.

[5] Braverman, M. and Kappeler, T. Refined analytic torsion as an element of the determinant line. Geom. Topol. 11 (2007), 139-213. MR 2302591 (2008a:58031)

[6] Braverman, M. and Kappeler, T. Comparison of the refined analytic and the Burghelea-Haller torsions. Festival Yves Colin de Verdiere. Ann. Inst. Fourier (Grenoble) 57 (2007), no. 7, 2361-2387.

[7] Braverman, M. and Kappeler, T. Refined analytic torsion. J. Differential Geom. 78 (2008), no. 2, 193-267.

[8] Braverman, M. and Kappeler, T. A canonical quadratic form on the determinant line of a flat vector bundle. Int. Math. Res. Not. IMRN 2008, no. 11, 21 pp.

[9] Braverman, M. and Vertman, B., A new proof of a Bismut-Zhang formula for some class of representations, Geometric and Spectral Analysis, Contemp. Math., vol. 630, Amer. Math. Soc., Providence, RI, 2014, pp. 1-14.

[10] Brüning, J. and Lesch, M. Hilbert complexes J. Funct. An. 108 88-132 (1992)

[11] Brüning, J. and Ma, X. An anomaly formula for Ray-Singer metrics on manifolds with boundary. Geom. Funct. Anal. 16 (2006), no. 4, 767-837.

[12] Brüning, J. and Ma, X. On the gluing formula for the analytic torsion, Math. Z. 273 (2013), no. 3-4, 1085-1117.

[13] D. Burghelea, L. Friedlander, and T. Kappeler, Meyer-Vietoris type formula for determinants of elliptic differential operators, Journal of Funct. Anal. 107 (1992), 34-65.

[14] Burghelea, D. and Haller, S., Euler Structures, the Variety of Representations and the Milnor-Turaev Torsion, Geom. Topol. 10 (2006), 1185-1238.

[15] Burghelea, D. and Haller, S., Complex-valued Ray-Singer torsion. J. Funct. Anal. 248 (2007), no. 1, 27-78.

[16] Burghelea, D. and Haller, S., Complex valued Ray-Singer torsion II. Math. Nachr. 283 (2010), no. 10, 1372-1402. 
[17] Cheeger, J. Analytic torsion and the heat equation. Ann. of Math. (2) 109 (1979), no. 2, 259-322.

[18] Farber, M. and Turaev, V. Absolute torsion. Tel Aviv Topology Conference: Rothenberg Festschrift (1998), 73-85, Contemp. Math., 231, Amer. Math. Soc., Providence, RI, 1999.

[19] Farber, M. and Turaev, V. Poincare-Reidemeister metric, Euler structures, and torsion. J. Reine Angew. Math. 520 (2000), 195-225.

[20] Farber, M. Absolute torsion and eta-invariant. Math. Z. 234 (2000), no. 2, 339-349.

[21] Farber, M. and Turaev, V., Poincaré-Reidemeister metric, Euler structures, and torsion, J. Reine Angew. Math. 520 (2000), 195-225.

[22] Gilkey, P. "Invariance Theory, the Heat-equation and the Atiyah-Singer Index Theorem", Second Edition, CRC Press (1995)

[23] Goldman, W. and Millson, J., The deformation theory of representations of fundamental groups of compact Kähler manifolds, Inst. Hautes Études Sci. Publ. Math. (1988), no. 67, 43-96.

[24] R. C. Gunning, Lectures on complex analytic varieties: The local parametrization theorem, Mathematical Notes, Princeton University Press, Princeton, N.J.; University of Tokyo Press, Tokyo, 1970. MR 0273060 (42 \#7941)

[25] R. C. Gunning, Lectures on complex analytic varieties: finite analytic mappings, Princeton University Press, Princeton, N.J.; University of Tokyo Press, Tokyo, 1974, Mathematical Notes, No. 14. MR 0355093 (50 \#7570)

[26] N.-K. Ho, The real locus of an involution map on the moduli space of flat connections on a Riemann surface, Int. Math. Res. Not. (2004), no. 61, 32633285 .

[27] Hörmander, L., An introduction to complex analysis in several variables, third ed., North-Holland Mathematical Library, vol. 7, North-Holland Publishing Co., Amsterdam, 1990.

[28] F. Kamber and Ph. Tondeur, Flat bundles and characteristic classes of grouprepresentations, Amer. J. Math. 89 (1967), 857-886.

[29] Kato, T. Perturbation Theory for Linear Operators, Die Grundlehren der math. Wiss. Volume 132, Springer (1966)

[30] Lee, Y. and Huang, R.-T. The refined analytic torsion and a well-posed boundary condition for the odd signature operator, arXiv:1004.1753v1 [math.DG]

[31] Lee, Y. and Huang, R.-T. The gluing formula of the refined analytic torsion for an acyclic Hermitian connection, Manuscripta Math. 139 (2012), no. 1-2, 91-122.

[32] Lee, Y. and Huang, R.-T. The comparison of two constructions of the refined analytic torsion on compact manifolds with boundary, J. Geom. Phys. 76 (2014), 79-96.

[33] Lesch, M. A gluing formula for the analytic torsion on singular spaces, Anal. PDE 6 (2013), no. 1, 221-256.

[34] Lück, W. Analytic and topological torsion for manifolds with boundary and symmetry. J. Differential Geom. 37 (1993), no. 2, 263-322.

[35] Müller, W. Analytic torsion and R-torsion of Riemannian manifolds. Adv. in Math. 28, (1978), no. 3, 233-305.

[36] Müller, W. Analytic torsion and R-torsion for unimodular representations. J. Amer. Math. Soc. 6 (1993), no. 3, 721-753. 
[37] L. Paquet Probl'emes mixtes pour le syst'eme de Maxwell, Annales Facult. des Sciences Toulouse, Volume IV, 103-141 (1982)

[38] Ray, D. B. and Singer, I. M. R-torsion and the Laplacian on Riemannian manifolds. Advances in Math. 7, 145-210. (1971).

[39] Shabat, B. V., Introduction to complex analysis. Part II, Translations of Mathematical Monographs, vol. 110, American Mathematical Society, Providence, RI, 1992, Functions of several variables, Translated from the third (1985) Russian edition by J. S. Joel.

[40] M. A. Shubin, Pseudodifferential operators and spectral theory, Springer Verlag, Berlin, New York, 1987.

[41] Turaev, V. G., Reidemeister torsion in knot theory, Russian Math. Survey 41 (1986), 119-182.

[42] Turaev, V. G., Euler structures, nonsingular vector fields, and Reidemeistertype torsions, Math. USSR Izvestia 34 (1990), 627-662.

[43] Turaev, V. G., Introduction to combinatorial torsions, Lectures in Mathematics ETH Zürich, Birkhäuser Verlag, Basel, 2001, Notes taken by Felix Schlenk.

[44] Vertman, B. Refined analytic torsion on manifolds with boundary. Geom. Topol. 13 (2009), no. 4, 1989-2027.

[45] B. Vertman, Gluing formula for refined analytic torsion, 2008, arXiv:0808.0451v2.

[46] Vishik, S. M. Generalized Ray-Singer conjecture. I. A manifold with a smooth boundary. Comm. Math. Phys. 167 (1995), no. 1, 1-102.

Department of Mathematics, Northeastern University,

Boston, MA 02115, USA

E-mail address: maximbraverman@neu.edu

$U R L$ : www.math.neu.edu/ ${ }^{\text {braverman/ }}$

Mathematisches Institut, Universität Bonn,

53115 Bonn, Germany

E-mail address: vertman@math.uni-bonn.de

$U R L$ : www math.uni-bonn.de/people/vertman 